\title{
A MULTIPLE SCALE MODEL FOR TUMOR GROWTH*
}

\author{
T. ALARCÓN ${ }^{\dagger}$, H. M. BYRNE ${ }^{\ddagger}$, AND P. K. MAINI ${ }^{\S}$
}

\begin{abstract}
We present a physiologically structured lattice model for vascular tumor growth which accounts for blood flow and structural adaptation of the vasculature, transport of oxygen, interaction between cancerous and normal tissue, cell division, apoptosis, vascular endothelial growth factor release, and the coupling between these processes. Simulations of the model are used to investigate the effects of nutrient heterogeneity, growth and invasion of cancerous tissue, and emergent growth laws.
\end{abstract}

Key words. blood flow heterogeneity, growth laws, physiologically structured lattice model, cell-cycle, p27, p53, multiple scales

AMS subject classification. 92B05

DOI. $10.1137 / 040603760$

1. Introduction. Cancer is believed to be responsible for one of every five deaths in Western countries and therefore is among the major killers in the developed world. The set of diseases categorized under this heading are characterized by major disruptions in the control mechanisms regulating growth and homeostasis in normal tissue [6]. In spite of the huge amount of resources that have been devoted to cancer research, many aspects remain obscure for experimentalists and clinicians, and many of the currently used therapeutic strategies are not entirely effective. The more systematic approach of mathematical modeling, used as a guide to biologists and clinicians, might help to elucidate the fundamental mechanisms of cancer progression and either to improve the therapeutic techniques currently used or to stimulate the development of new strategies.

In fact, modeling cancer has become one of the most active areas within the mathematical and theoretical biology communities. Many models have been proposed focusing on different aspects. Broadly speaking, we can divide these into two approaches: continuum models, mathematically formulated in terms of partial differential equations (PDEs), and cellular automaton (CA) models.

Typically, tumor growth is divided into three stages: avascular growth, angiogenesis, and vascular growth. In the earliest stage, the avascular stage, the tumor develops in the absence of blood supply (hence the name). In this first stage typically the tumor grows up to a maximum size, since its growth is limited by the amount of nutrients the tumor can obtain through its surface [22]. In the second stage, some of the cells of this avascular tumor mass produce and release substances called tumor angiogenic factors (TAFs). TAF diffuses through the surrounding tissue, and, upon

${ }^{*}$ Received by the editors February 3, 2004; accepted for publication (in revised form) June 17, 2004; published electronically February 9, 2005.

http://www.siam.org/journals/mms/3-2/60376.html

†Bioinformatics Unit, Department of Computer Science, University College London, Gower Street, London WC1E 6BT, UK (t.alarcon@cs.ucl.ac.uk). This author's work was supported by the EU Research Training Network (5th Framework): "Using mathematical modelling and computer simulation to improve cancer therapy."

${ }^{\ddagger}$ Centre for Mathematical Medicine, School of Mathematical Sciences, University of Nottingham, Nottingham NG7 2RD, UK (helen.byrne@maths.nottingham.ac.uk). This author's work was supported by the EPSRC as an Advanced Research Fellow.

$\S$ Centre for Mathematical Biology, Mathematical Institute, University of Oxford, 24-29 St Giles', Oxford OX1 3LB, UK (maini@maths.ox.ac.uk). 
arrival to the vasculature, it triggers a cascade of events (angiogenesis) which eventually leads to vascular growth towards the tumor. Once vascularization is completed, the tumor enters the vascular stage. In this stage, the tumor has access to virtually unlimited resources, so it can grow beyond its limited "avascular" size, and, also, it acquires a means of transport for cells that intravasate into the vasculature and form metastases in any part of the host organism. Thus, whereas in the avascular phase tumors are basically harmless, once they become vascular they are potentially fatal.

Among the continuum models, Greenspan proposed some of the earliest mathematical models of tumor growth [33,34]. His models of avascular tumor growth were formulated as moving boundary problems, in which the solid tumor grows in suspension. The models do not allow for cellular heterogeneity within the tumor mass, and the treatment of the mechanical properties of the tissue is rather simplistic. For extensions of Greenspan's moving boundary formulation see [1, 2, 12, 47].

Significant progress was made with the introduction of multiphase models. These models extend the moving boundary approach to incorporate cellular heterogeneity and the use of more complex mechanical laws to describe the response of the tissue to external forces. Multiphase models have now been used to model avascular growth [13, 54, 71, 72], vascular growth [10], ductal carcinoma in situ [23], and tumor encapsulation [38].

The approaches described above draw upon methods from fluid and continuum mechanics. Models based on different approaches have been successful in describing other aspects of tumor growth. One approach involves using reaction-diffusion theory as a modeling framework. Such models have been used to describe invasion and different aspects of tumor-induced angiogenesis [15, 45, 46, 51]. An alternative approach which has been used to model interactions between tumor growth and the immune system is based on kinetic theory and Boltzmann-like equations [9]. For a more extensive review of continuum modeling of tumor growth see [57].

Whereas continuum models describe cell populations by means of continuous fields, cellular automata (CAs) deal with the dynamics of discrete elements. ${ }^{1}$ These elements take their state from a discrete (finite) space of states and evolve in discrete space and time. The dynamics of the elements is given in terms of local rules (either deterministic or probabilistic). Some of the models we describe below incorporate modifications to the classical definition of a CA. In particular, some of them introduce diffusive substances (such as nutrients or signaling cues) which are described by means of continuum fields. These models are categorized as hybrid CAs and are the basis for the development of the multiple scale models we discuss in this paper. CA models have been proposed to describe four aspects of tumor growth, namely, avascular growth, vascular growth, invasion, and angiogenesis.

The model proposed in [20] is formulated as a two-dimensional hybrid CA (or, more precisely, a lattice-gas model [63]) and reproduces many of the features of avascular tumors in vitro, as, for example, their layer structure.

In [52], a hybrid CA model of tumor growth in the presence of native vasculature is proposed ${ }^{2}$ to analyze the role of host vascular density and tumor metabolism on tumor growth. Tumor cells seem to use the glycolytic metabolism pathway rather

\footnotetext{
${ }^{1}$ In the biological context these elements might be either individual cells or (small) clusters of cells.

${ }^{2}$ Neither interaction between colony development and vasculature nor angiogenesis is taken into account.
} 
than the aerobic metabolism, as normal cells do. One by-product of glycolisis is the increase of acidity. Since tumor cells are more resistant to acidity than their normal counterparts, it appears that cancer uses the glycolytic phenotype in order to increase its invasiveness [29]. Several results regarding the interplay between vessel density, increased acidity, and tumor progression are obtained. Probably the most significant results are the presence of a sharp transition between states of initial tumor confinement and efficient invasiveness when $\mathrm{H}^{+}$production passes through a critical value. This has been observed in experiments [29].

In [68] malignant invasion using the extended Potts model [32] is studied. The authors investigate how malignant cell phenotypes, in particular increased adhesion, affect cancer invasiveness. Their extended Potts model is based on the minimization of an energy function by a Monte Carlo method. The model can be simulated to investigate how the maximum depth of invasion changes as different parameters relating to cell-to-cell and cell-to-extracellular matrix adhesion and haptotaxis are varied.

A CA model for angiogenesis based on endothelial cell migration in response to gradients of TAF (chemotaxis) and fibronectin ${ }^{3}$ (haptotaxis) is proposed in [7]. In this model vessel tip migration is modeled by a biased random walk derived from finitedifference discretizations of macroscopic level PDEs. Additional rules are provided for branching and anastomosis. ${ }^{4}$ The results can reproduce experimental observations on solid tumor implants in the cornea of animals [7].

For more extensive reviews of CA modeling of biological systems in general and tumor growth in particular see [5] and [48], respectively.

In spite of all this effort, much work remains to be done in order to produce clinically relevant and predictive models. One obstacle that must be overcome is the intrinsic multiple scale nature of tumor growth. It involves processes occurring over a variety of time and length scales: from the tissue scale (for example, vascular remodeling) to intracellular processes (for example, progression through the cell-cycle). The structure of a solid, vascularized tumor is very dynamic and heterogeneous. Within a solid tumor a given region may, at some point, become hypoxic. This situation triggers biochemical pathways within the cancer cells involved in a number of intracellular responses to hypoxia (vascular endothelial growth factor (VEGF) secretion and release, transition to quiescence, etc.). VEGF release by starving cells triggers angiogenesis (i.e., recruitment of new vasculature from the existing one), which results in the supply of oxygen to the hypoxic regions. Upon successful angiogenesis, cells do not lack oxygen anymore and emerge from quiescence into the proliferating state. However, tumor vessels are less stable than their normal counterparts and when coopted by the growing cancerous tissue they undergo a dematuration process. As a consequence, these newly formed vessels collapse very easily, and the whole process starts all over again. This is just an illustrative example of how processes occurring at very different time and length scales (TLSs) are coupled.

Most of the models described so far, with the exception of those presented in [20] and [52], focus on one scale. While they may provide valuable insight into processes occurring at that scale, they do not address the fundamental problem of how phenomena at different scales are coupled. In this article, we aim to describe a framework for formulating a multiple scale model of tumor growth capable of integrating a hierarchy of processes occurring at different scales.

\footnotetext{
${ }^{3}$ Fibronectin is a molecule that enhances cell adhesion to the extracellular matrix. It is a component of the extracellular matrix and is also expressed by the endothelial cells.

${ }^{4}$ Anastomosis is the formation of a loop by fusion of two capillaries.
} 
MULTISCALE MODELING OF TUMOR GROWTH

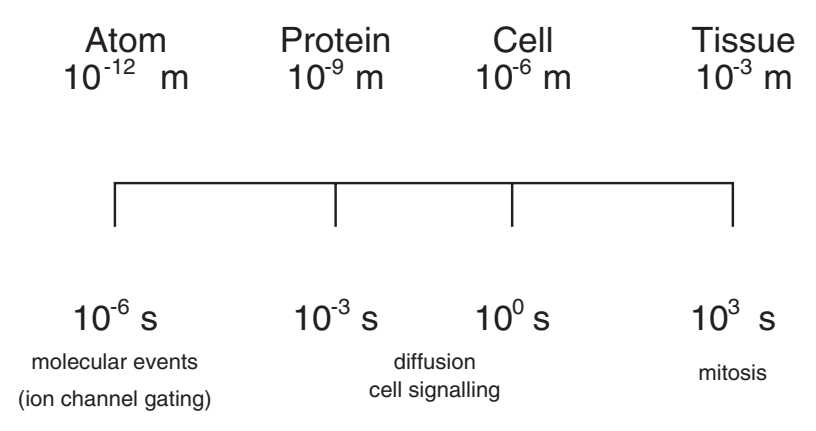

FIG. 1. TLSs involved in our model [36].

Our aim in this work is to extend our previously developed modeling framework [3] to include phenomena occurring on a wide range of TLSs and the coupling between them (see Figure 1).

Similar modeling approaches that integrate different TLSs have been proposed to describe other biological processes. For example, in physiology, integrative modeling of the heart is a particularly active area of research $[55,66,37]$. In developmental biology, the formation of the skeletal pattern in the avian limb bud has been simulated by means of a scheme aimed to integrate cell-to-cell interactions, intracellular processes, and domain-level reaction-diffusion models of genetic regulation [16]. Physiologically structured models to describe predator-prey systems under a distribution of nutrients have been proposed [41]. In the particular area of tumor growth a model combining progression through the cell-cycle and cell migration has been recently proposed in [53].

This article is structured as follows. In section 2, we present a general description of the model, focusing on its general structure and explaining how the different elements (corresponding to different TLSs) are coupled together. The next three sections are devoted to introducing the different elements we include in our model: the blood flow, the dynamics for the vascular structural adaptation, and the oxygen transport into the tissue (section 3), the competition between cancer and normal cells (section 4), and the dynamics of the intracellular processes (cell division, apoptosis, VEGF secretion) (section 5). Section 6 contains the rules of our lattice model and incorporates all the elements detailed in sections 3 , 4, and 5 . In section 7 , we discuss the growth and invasion patterns our model yields in different conditions and some predictions that may be of interest from the point of view of therapy. In section 8, we give an account of the growth laws that our model predicts in different situations. Finally, in section 9, we summarize our results and discuss the limitations of the model together with future research directions.

2. General structure of the model. The model we present in this article integrates phenomena occurring on very different TLSs (see Figure 1). These features include blood flow and structural adaptation of the vascular network, transport into the tissue of bloodborne oxygen, competition between cancer and normal cells, cell division, apoptosis, VEGF release, and the coupling between them. Its structure, therefore, is quite complex. For this reason, before presenting the submodels we use to describe the different phenomena, we devote this section to explaining the overall structure of the modeling framework.

The modeling framework we use is an extension of the hybrid cellular automaton, 


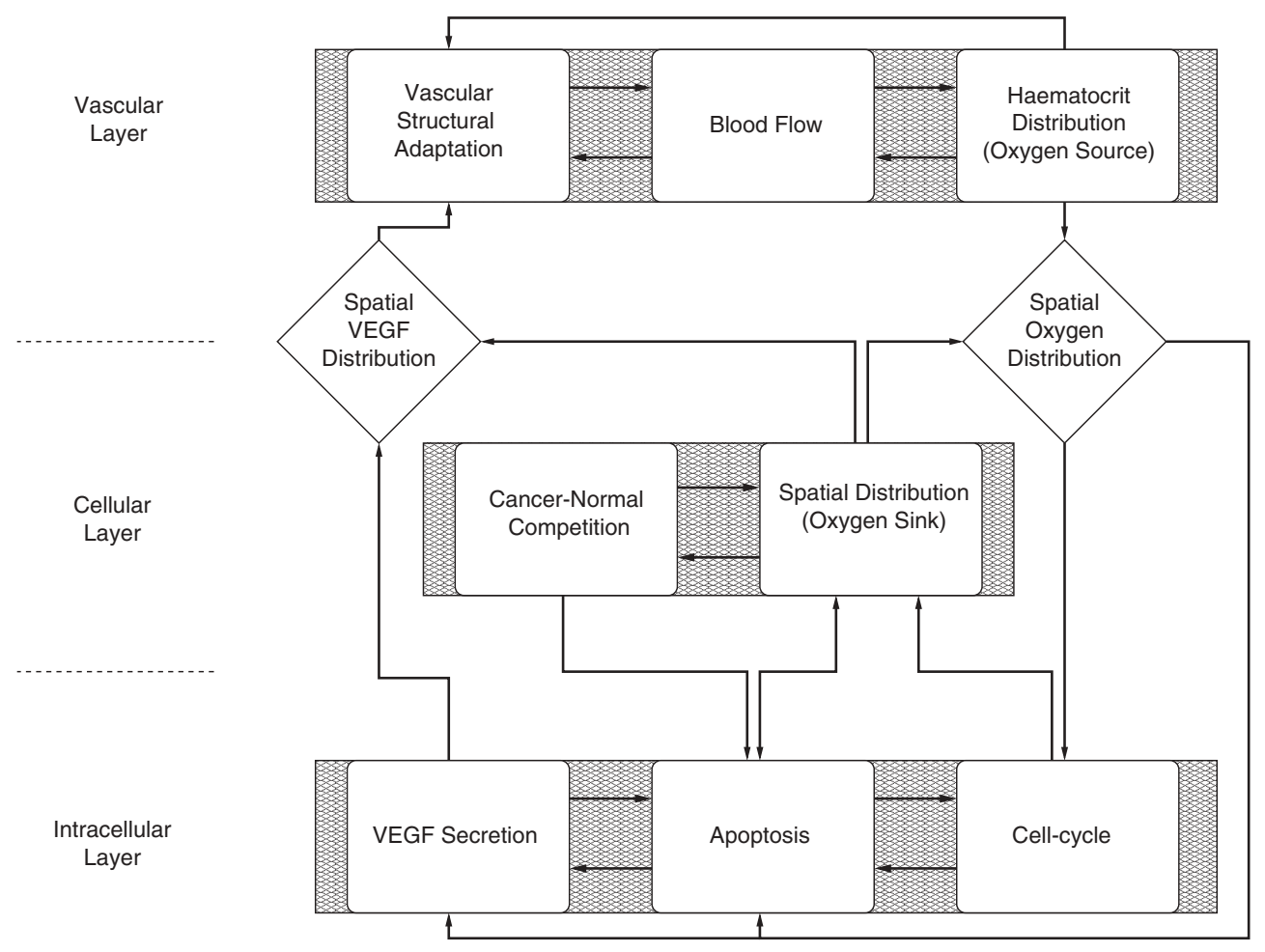

FIG. 2. Diagrammatic representation of the layer structure of our model.

which has been used to model several aspects of tumor development (see [52, 20, $3]$ ). Here we extend this concept to account not only for the presence of a diffusive substance (such as oxygen or glucose) as in previous works but also for intracellular and tissue-scale phenomena and the coupling between them. To this end, we have organized our model in three layers: the vascular, the cellular, and the intracellular layers, which correspond to the tissue, cellular, and intracellular TLSs, respectively (see Figure 2).

In the top layer, we deal with the structure of the vascular network and blood flow (see section 3 and [3] for more details). We consider a hexagonal vascular network (similar to the one observed in liver; see [44]). Each individual vessel is assumed to undergo structural adaptation (i.e., changes in radius) in response to different stimuli until the network reaches a stationary state. Through this structural adaptation process we compute the blood flow rate, the pressure drop, and the haematocrit (i.e., relative volume of red blood cells) in each vessel, thus providing the distribution of haematocrit over the vascular network. Between the vascular layer and the cellular layer, i.e., coupling the dynamics at the cellular level to blood flow and vascular adaptation, we have the transport of bloodborne oxygen into the tissue. This process is modeled by means of a reaction-diffusion equation. The distribution of haematocrit is the source of oxygen, whereas the distribution of cells (provided by the cellular layer) gives us the (spatially distributed) sink of oxygen. We give a full account of the modeling in the vascular layer in section 3.

In the intermediate layer, we focus on cell-cell interactions (competition) and spatial distribution of cells. We consider two types of cells: normal and cancer cells, which 
are modeled as individuals (see section 4 for more details). These two populations compete for space and resources. Cancerous phenotypes are usually better competitors, which result in the cancer population taking over. One of the aims of this paper is to identify the key model parameters which may allow this situation to be reversed. In section 7, we propose one such mechanism. Competition between the two types of cells is introduced by a very simple rule, which, in turn, couples this middle layer with the intracellular layer. Apoptosis (programmed cell death) is controlled by the expression of p53 (whose dynamics is dealt with in the intracellular layer): when the level of p53 in a cell exceeds some threshold the cell undergoes apoptosis. However, this threshold is fixed according to the local spatial distribution of cells, which links the spatial distribution (cellular layer) with the apoptotic process (intracellular layer). More details of all the rules involved in the cellular dynamics, in particular how these thresholds are fixed, are given in section 4.

In the bottom layer, we focus on intracellular processes, in particular cell division, apoptosis, and VEGF secretion. In this layer, we use ordinary differential equations (ODEs) to model the relevant biochemistry. One issue we focus on is how the external conditions modulate the dynamics of these intracellular phenomena and, in particular, how the level of extracellular oxygen affects the division rate or the expression of p53 (which regulates apoptosis) and the production of VEGF. Since the spatial distribution of oxygen depends on both the spatial distribution of cells (cellular layer) and on the distribution of haematocrit (vascular layer), these processes at the intracellular level are linked to the behavior of the other two layers: cell proliferation and apoptosis alter the spatial distribution of the cells (see Figure 2); the cellular and the intracellular layers modulate the process of vascular structural adaptation through another transport process: diffusion of VEGF into the tissue and its absorption by the endothelial cells lining the vessels (see section 3). A full account of the ODE models used is given in section 5 .

From a more formal point of view, the introduction of the intracellular layer means that our model actually fails to fall into the category of a CA model. The definition of a CA implies a discrete-time description of the dynamics of the system [48], whereas the inclusion of the ODE models for cell division and other intracellular processes yields a continuous-time description. So, rather than a CA model, our model might be better categorized as a physiologically structured lattice model.

3. Vascular structural adaptation and blood flow. In this section we give details of the elements we have included in our blood flow simulations. As in [3], we take into account three major factors, namely, vascular structural adaptation, complex blood rheology, and the red blood cell distribution at bifurcations. However, here we incorporate a new element: the coupling between the metabolic needs of the tissue and the vascular structural adaptation mechanism.

Figure 3 shows a schematic representation of the vascular network we use for our simulations. We consider a vascular hexagonal network, similar to those usually found, for example, in the liver [44]. Although the particular geometry of the vascular network might have an effect on the pattern of growth predicted by our model, a thorough investigation of the effect of different vascular geometries on growth is beyond the scope of this paper. We have chosen this particular geometry because it has been observed in real systems, and hence it enables us to show that even in a highly regular geometry, the complexities of blood flow and vascular dynamics may give rise to complex behavior. In Figure 3(a) we see that our hexagonal vascular network is superimposed on a rectangular grid, $\mathcal{G} \subset \mathbb{Z}^{2}$. A point in this grid is denoted by the 

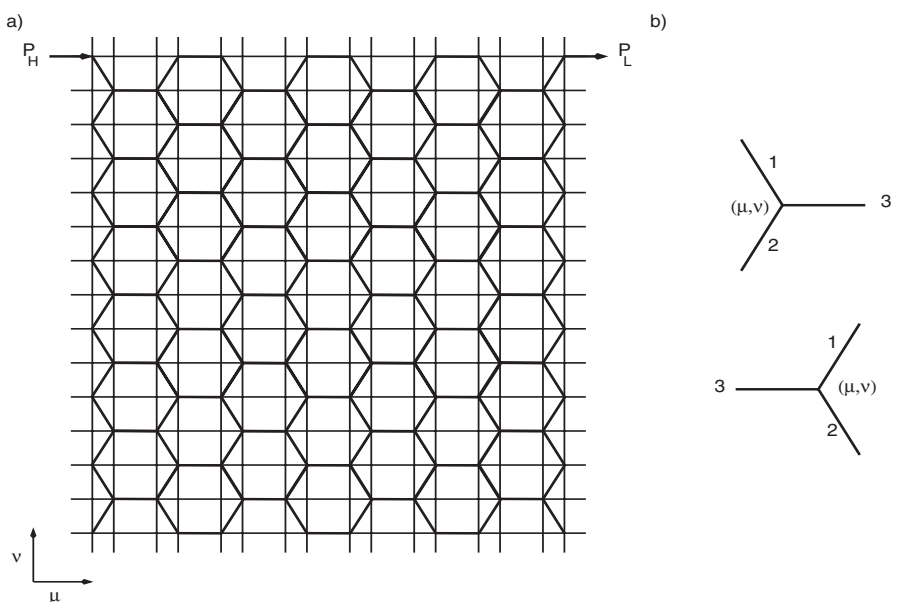

FiG. 3. Diagrammatic representation of the layer structure of our vasculature network model.

pair $(\mu, \nu) ; \mu, \nu \in \mathbb{Z}$. On this grid we define the quantity $N(\mu, \nu)$ by

$$
N(\mu, \nu)=\left\{\begin{array}{l}
1 \text { if }(\mu, \nu) \text { is a vertex of the vascular network } \\
0 \text { otherwise. }
\end{array}\right.
$$

We assume that the flow in each vessel may be characterized by Poiseuille's law [24]. Since Poiseuille's law implies that the pressure drop in each vessel is proportional to the flow rate, we can use Kirchoff's laws to compute the flow rate, $\dot{Q}$, the pressure drop, $\Delta P$, the pressure, $P$, the wall shear stress, $\tau_{w}$, and the haematocrit, $H$, in each vessel of our network [3].

We label each vessel using the numbering scheme shown in Figure 3(b). If, for example, we want to refer to the radii of some of the vessels meeting at a point of $\mathcal{G}$ at time $t$, we use the notation $R_{i}(\mu, \nu ; t) ; i=1,2,3$. Of course, $N(\mu, \nu)=1$ at such a point. Similar notation is used for the other hydrodynamic quantities.

The initial condition for the vascular adaptation algorithm is a configuration in which all the vessels have the same radius $R_{0}$ so that

$$
R_{i}(\mu, \nu ; t=0)=R_{0} \quad \forall \mu, \nu, i .
$$

The remaining hydrodynamic quantities are initially set to zero. As shown in Figure 3(a), a pressure drop $\Delta P_{T}=P_{H}-P_{L}$ is imposed between the two top corners of the network. No flux conditions are imposed elsewhere on the boundary. $\Delta P_{T}$ is kept constant throughout the simulation. Given this initial configuration, we use Kirchoff's laws to calculate the hydrodynamic quantities in each vessel. This then provides us with initial conditions for the structural adaptation algorithm [3].

The vascular system is continually influenced by the flow and the demands of the surrounding tissue and remodels itself accordingly. Pries et al. formulated an adaptation mechanism which describes how the lumen radius, $R_{i}(\mu, \nu ; t)$, is modified by three such stimuli [60]. The first stimulus that they considered was mechanical. In particular, Pries et al. proposed that vascular networks adapt themselves in order to maintain a fixed relationship between transmural pressure and wall shear stress (hydrodynamic stimulus) [59] (see (3.4)). Structural adaptation of vascular beds also occurs in response to the metabolic demands of the surrounding tissue. Consequently, 
if the flow in some vessels drops and the tissue becomes poorly supplied with oxygen or other metabolites, then the vasculature will be stimulated to grow. The third stimulus is the so-called shrinking tendency, which accounts for the tendency of the radii of the vessels to decrease in the absence of growth factors that maintain or increase the size of a given vessel. ${ }^{5}$ In [60] Pries et al. modeled the effect that these stimuli have on the vessel radii by using the following equation:

$$
\begin{aligned}
R_{i}(\mu, \nu ; t+\Delta t)= & R_{i}(\mu, \nu ; t)+R_{i}(\mu, \nu ; t) \Delta t\left(\log \left(\frac{\tau_{w i}(\mu, \nu ; t)}{\tau\left(P_{i}(\mu, \nu ; t)\right)}\right)\right. \\
& \left.+k_{m}\left(V_{i}(\mu, \nu ; t)\right) \log \left(\frac{\dot{Q}_{r e f}}{\dot{Q}_{i}(\mu, \nu ; t) H_{i}(\mu, \nu ; t)}+1\right)-k_{s}\right) .
\end{aligned}
$$

In (3.3) $\Delta t$ is the time step, $\dot{Q}_{r e f}$ is the minimum flow rate required to maintain tissue homeostasis, and $K_{m}$ and $k_{s}$ are constants accounting for the intensity of the metabolic stimulus and shrinking tendency, respectively [60]. For Poiseuille flow, $\tau_{w}=4 \mu \dot{Q} / \pi R^{3}$. The first, second, and third terms on the right-hand side of (3.3) correspond to the hydrodynamic, metabolic, and shrinking tendency stimuli, respectively. $\tau\left(P_{i}(\mu, \nu ; t)\right)$ is the wall shear stress-pressure relationship that the vascular system attempts to maintain. The expression for $\tau\left(P_{i}(\mu, \nu ; t)\right)$ that we use was obtained in [60] by fitting to data from the rat mesentery and is given by

$$
\tau\left(P_{i}(\mu, \nu ; t)\right)=100-86\left[\exp \left(-5000 \log \left(\log P_{i}(\mu, \nu ; t)\right)\right)\right]^{5.4} .
$$

We have introduced a modification in the original formulation of the structural adaptation mechanism. In the third term on the right-hand side of (3.3) we have introduced a function $k_{m}(V)$. In the original formulation $k_{m}$ was a constant. Here we have introduced a dependence of this parameter on the concentration of VEGF $V$. VEGF is produced by many types of cells, including tumor cells, as a response to hypoxia. VEGF stimulates proliferation of the endothelial cells lining the vessels. Thus by assuming $k_{m}=k_{m}(V)$ we model directly the interaction between the surrounding tissue and the vasculature, rather than the effective interaction introduced in [60]. When the tissue is oxygen-starved, VEGF is produced and released. This changes the structure of the vascular network and the oxygen distribution, which may, in turn, reduce the rate of production of VEGF. In sections 4 and 5, we explain how our model cells produce and release VEGF and how it diffuses through the tissue.

A more realistic way of modeling the interaction between the tissue and vasculature would be to introduce angiogenesis, i.e., recruitment of new blood vessels in response to the angiogenic stimulus (i.e., VEGF). Rather than explicitly introducing new vessels that sprout from the existing vasculature, we simply increase the radius of the existing vessels. Basically we are replacing a complex network of vessels by a single effective cylindrical vessel of an appropriate radius. This approximation is used extensively in plant sciences to model nutrient uptake: the complex tree-like root structure is modeled by a single cylinder of an equivalent radius $[8,50,62]$. The angiogenic response in our model, therefore, leads to an increase in the radii of the vessels. In particular, we assume that the intensity of the metabolic stimulus in (3.3) is modulated by the local concentration of VEGF. The particular expression we use

\footnotetext{
${ }^{5}$ In [60] other stimuli were considered. We have considered only these three, since they provide the simplest stable adaptation mechanism.
} 
to implement this interaction is

$$
k_{m}(V)=k_{m}^{0}\left(1+\frac{V}{V_{0}+V}\right) .
$$

Another factor we consider is blood rheology. Blood is not a simple fluid with constant viscosity: it is a complex suspension of cells and molecules of a wide range of sizes. Thus, modeling blood as a Newtonian fluid is a crude approximation. On the other hand, trying to tackle the problem of blood rheology in all its complexity would be an overwhelming task. Hence, as in [3], we focus only on the effect of the red blood cells (RBCs), as they seem to play a major role in blood flow [24].

Due to the presence of RBCs the blood viscosity depends on the haematocrit $(H)$ and on the radius $(R)$. For a given $H$, the viscosity depends nonmonotonically on $R$, and three different flow regimes may be identified. If $R$ is much greater than the typical size of a RBC, ${ }^{6}$ the viscosity is independent of $R$. As $R$ decreases, the viscosity also decreases (the Fahraeus-Lindqvist effect). This behavior persists until the vessel radius is of the order of $15-20 \mu \mathrm{m}$. The viscosity then reaches a minimum and, thereafter, increases if $R$ is similar in magnitude to the radius of a RBC [58]. Pries et al. have fitted the following explicit expression for the viscosity as a function of $R$ and $H$ to detailed experimental data [58]:

$$
\begin{aligned}
& \mu_{\text {blood }}=\mu_{\text {plasma }} \cdot \mu_{\text {rel }}, \\
& \mu_{\text {rel }}=\left[1+\left(\mu_{0.45}^{*}-1\right) \frac{(1-H)^{C}-1}{(1-0.45)^{C}-1}\left(\frac{2 R}{2 R-1.1}\right)^{2}\right]\left(\frac{2 R}{2 R-1.1}\right)^{2}, \\
& \mu_{0.45}^{*}=6 e^{-0.17 R}+3.2-2.44 e^{-0.06(2 R)^{0.645}}, \\
& C=\left(0.8+e^{-0.15 R}\right)\left(-1+\frac{1}{1+10^{-11}(2 R)^{12}}\right)+\frac{1}{1+10^{-11}(2 R)^{12}},
\end{aligned}
$$

where $\mu_{r e l}$ is the blood viscosity scaled by the viscosity of the plasma.

Since $\mu_{\text {rel }}$ depends on $H$, the haematocrit distribution is an important factor when determining the hydrodynamic state of the network. The simplest way to proceed is to assume that at each bifurcation the distribution of $H$ depends on the flow velocity in each of the daughter vessels [24]. Roughly speaking, a larger proportion of the haematocrit from the parent vessel is transported along the faster branch. Additionally, it has been observed in model experiments with rubber, RBC-shaped pellets that at bifurcations where the ratio between the velocities of the branches exceeds a certain threshold, all of the haematocrit enters the faster branch. Combining these results we assume that, at a bifurcation, the haematocrit is distributed as follows:

$$
\begin{aligned}
& H_{p}=H_{1}+H_{2} ; \\
& \frac{H_{1}}{H_{2}}=\alpha \frac{v_{1}}{v_{2}}, \text { if } \frac{v_{1}}{v_{2}}<T H R ; \\
& H_{1}=H_{p}, \text { if } \frac{v_{1}}{v_{2}}>T H R,
\end{aligned}
$$

where $H_{p}$ is the haematocrit in the parent vessel, $H_{1}$ and $H_{2}$ are the haematocrits in the daughter vessels, $v_{i}(i=1,2)$ are the average flow velocities on a section orthogonal to the axis of the daughter vessels and are defined by $v_{i} \equiv \dot{Q}_{i} / \pi R_{i}^{2}, \alpha$ is a

\footnotetext{
${ }^{6}$ The average $\mathrm{RBC}$ diameter in humans is $7-8 \mu \mathrm{m}$.
} 
(a)

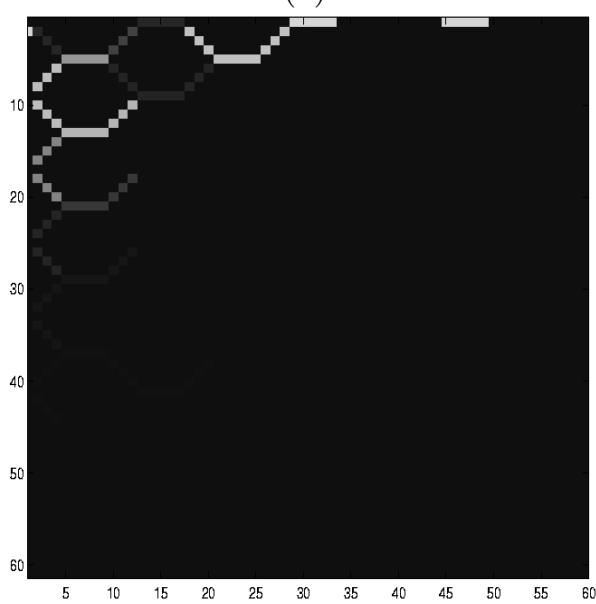

(b)

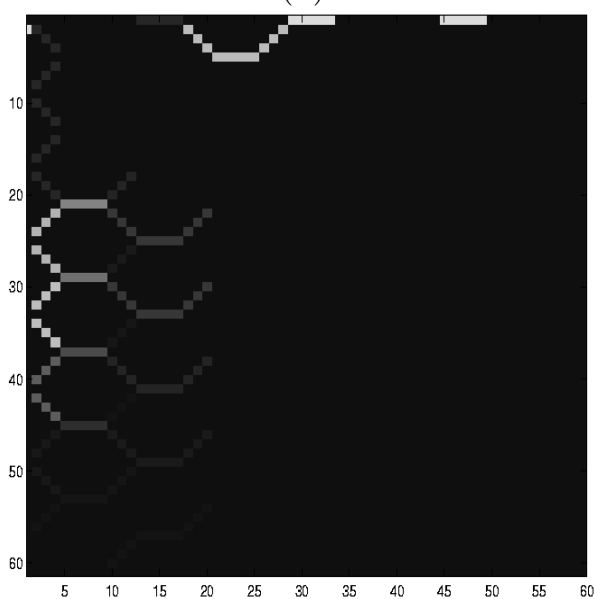

FIG. 4. (a) Distribution of haematocrit in the vascular network without coupling between tissue and vasculature. (b) Distribution of haematocrit in the vascular network with coupling between vasculature and development of a colony of normal cells (see section 7.1 for details). In these figures the greyscale corresponds to the concentration of haematocrit: lighter grey corresponds to higher haematocrit levels.

phenomenological parameter which accounts for the strength of the asymmetry of the haematocrit distribution at bifurcations, and $T H R$ is the value of the ratio between the velocities of the branches above, where all of the haematocrit goes to the faster branch.

The features displayed by the viscosity and the behavior of RBCs at bifurcations give rise to a highly complex, nonlinear problem, in which the viscosity depends on the dynamical state of the entire vascular network, including the vessel radii. The vessel radii, in turn, depend on the hydrodynamic quantities through the adaptation mechanism [3].

Figure 4 shows results of our blood flow simulations, in particular of the distribution of haematocrit. Figure 4(a) corresponds to the case analyzed in [3], in which the evolution of the vasculature is independent of the tissue. Figure 4(b) shows the result for a case in which there is tissue-vasculature interaction through the aforementioned dependence of the metabolic stimulus on the VEGF concentration (see section 7.1 for details). We can see that the distribution of haematocrit is sparser in the second case, due to this interaction.

The process leading to this tissue-dependent vascular remodeling can be understood by looking at Figure 5. The three panels in the top row show the spatial distribution of cells (Figure 5(a)), the distribution of oxygen (Figure 5(b)), and the distribution of VEGF (Figure 5(c)), for an early stage of colony development. Figures $5(\mathrm{~d})$, (e), and (f) (middle row) show the same quantities for an intermediate stage of colony growth. Figures $5(\mathrm{~g}),(\mathrm{h})$, and (i) (bottom row) show the stationary distributions of these three quantities. Initially, oxygen (which corresponds to the distribution of haematocrit shown in Figure 4(a)) is concentrated in a localized region. Cells at a distance from this region are starved of oxygen and respond by producing VEGF (Figures 5(b) and (c)). In response to VEGF, the vasculature remodels itself, allowing more oxygen to reach those regions and allowing further growth of the colony (Figures $5(\mathrm{~d})$ and (e)). As a consequence of growth, oxygen is used up, and more cells become 
(a)

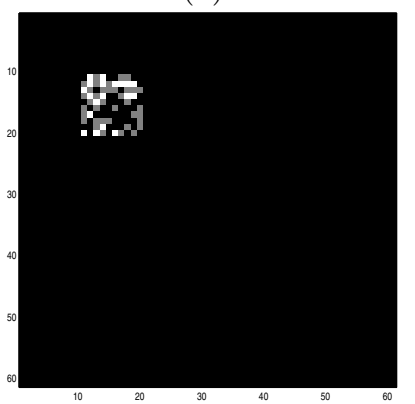

(d)

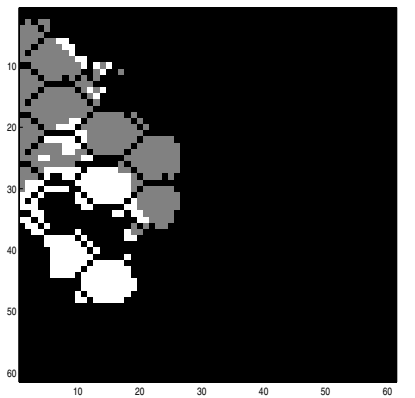

(g)

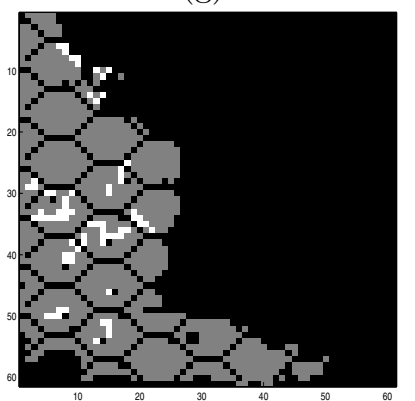

(b)

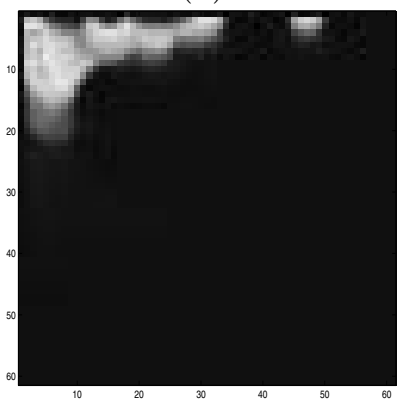

(e)

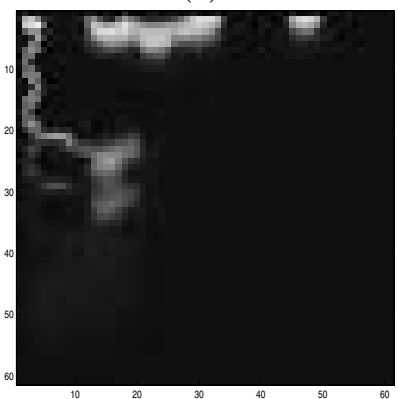

(h)

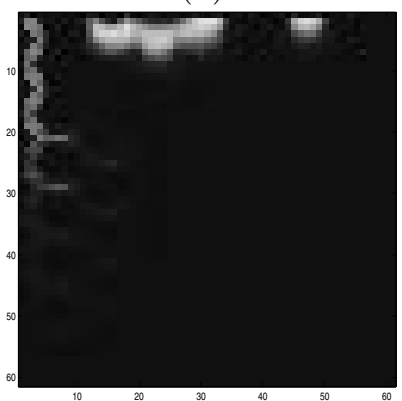

(c)

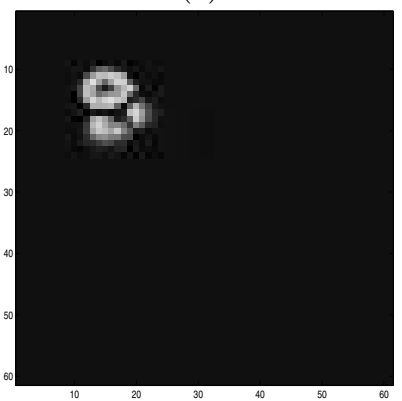

(f)

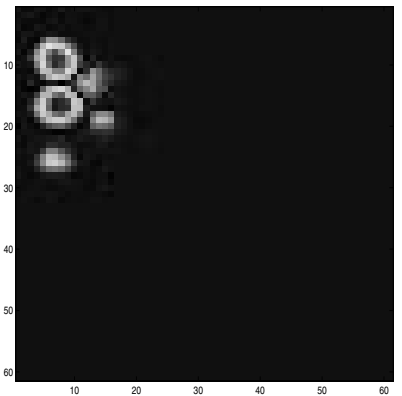

(i)

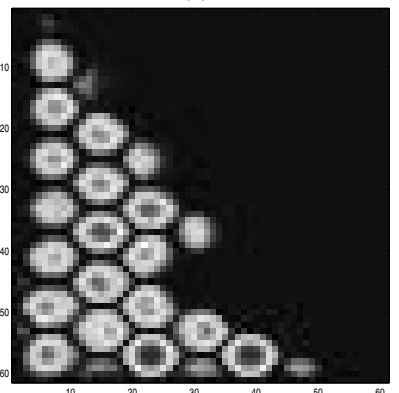

FIG. 5. Snapshots of the evolution of the colony of cells (left column), oxygen distribution (central column), and VEGF distribution (right column). In the central and right column figures the greyscale corresponds to the concentration of oxygen and VEGF, respectively: lighter grey corresponds to higher concentrations of oxygen or VEGF.

hypoxic and secrete VEGF (Figure 4(f)). This VEGF promotes further remodeling of the vascular network. Eventually, a stationary state is reached (Figures $5(\mathrm{~g})$, (h), and (i)) at which the colony attains the maximum size allowed by the amount of oxygen available.

4. Cellular dynamics. In this section we discuss the discrete dynamics used to model the cells. After providing the basic definitions and notation, we will give an account of how our cells interact with each other and how we formulate the different boundary value problems used to determine the distribution of oxygen and extracellular VEGF within the tissue domain.

We consider a square lattice, $\mathcal{L}=\mathbb{Z}^{2}$, consisting of a set of elements (nodes) labeled by their positions $\mathbf{r} \in \mathcal{L}$, with $\mathbf{r}=(i, j) i, j \in \mathbb{Z}$ (see Figure 6). With each 


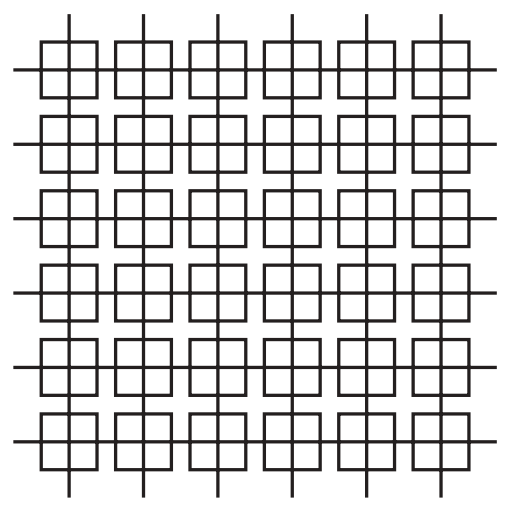

FIG. 6. Schematic representation of the lattice used in our simulations.

node of the lattice we associate a neighborhood. For our square lattice the nearest neighbors neighborhood is given by

$$
\begin{aligned}
& \mathcal{N}(\mathbf{r})=\left\{\mathbf{r}+c_{k} ; k=1, \ldots, b\right\}, \\
& c_{1}=(1,0), \\
& c_{2}=(0,1), \\
& c_{3}=(-1,0), \\
& c_{4}=(0,-1) .
\end{aligned}
$$

The constant $b$ is the coordination number of the lattice. In our case, $b=4$. The nearest neighbors neighborhood is basically determined by the geometry of the lattice.

Each element is characterized by a state $s(r)$ :

$$
\mathrm{s}(\mathrm{r}): \mathrm{r} \in \mathcal{L} \longrightarrow \mathcal{E}
$$

where $\mathcal{E}=\mathbb{Z}^{2} \times \mathbb{R}^{11}$ is our state space, which in the present case is 13-dimensional. The first component $s_{1}(r)$ corresponds to the type of cell occupying the element $r$ : it takes value 0 if it is empty, 1 if it is a cancer cell, 2 if it is a normal cell, and 3 if it is a vessel. The second component stores the number of divisions that the cell has undergone (see section 5). This is set to zero for an empty element or an element occupied by a vessel. Components 3 and 4 are for the local concentration of oxygen and extracellular VEGF. Components 5-9 store the concentration of the substances controlling the cell-cycle (see section 5.1). The last three components are occupied by the cell phase (see section 5.1), the local expression of p53, and the intracellular concentration of VEGF (see section 5.2).

We have incorporated the vessels (black elements) by mapping the vascular grid $\mathcal{G}$ onto $\mathcal{L}$. Starting with an empty lattice (i.e., $\mathrm{s}_{1}(\mathrm{r})=0$ for all $\mathrm{r}$ ) we have performed the following operation:

$$
\mathrm{s}_{1}(4(\mu-1)+1,4(\nu-1)+1)=3 \quad \forall(\mu, \nu) \in \mathcal{G} \text { such that } N(\mu, \nu)=1 .
$$

This operation maps the vertices of the vascular network onto $\mathcal{L}$. The next step is to fill the elements connecting the vertices by a straight line with vessel elements $\left(\mathrm{s}_{1}(\mathrm{r})=3\right)$. Likewise, we assign the values of the hydrodynamic and vascular quantities, in particular the haematocrit, which are associated with the corresponding vessel. 
Once we have mapped the distribution of haematocrit onto $\mathcal{L}$, we can solve the boundary value problem (BVP) for the oxygen as in [3]. Assuming that the relaxation time for the oxygen distribution is much shorter than the characteristic time scale for the evolution of the cells, we can write

$$
D_{P} \nabla^{2} P-k_{P}(\mathrm{r}) P=0 .
$$

The function $k(r)$, which is updated on each iteration of our automaton, depends on the cell distribution and is defined by

$$
k_{P}(\mathrm{r})=\left\{\begin{array}{l}
k_{N}^{P} \text { if there is a normal cell at } \mathrm{r}, \\
k_{C}^{P} \text { if there is a cancer cell at } \mathrm{r} \\
0 \text { otherwise }
\end{array}\right.
$$

It remains to prescribe appropriate boundary conditions for (4.4), which couple the dynamics of the tissue with the distribution of haematocrit in the vascular network. Oxygen enters the system by crossing the walls of the vessels, the flux being given by $\vec{J}=-D_{P} \nabla P$. Thus we impose the following mixed boundary conditions at the walls of the vessels:

$$
-D_{P} \mathbf{n}_{w} \cdot \nabla P=\mathcal{P}\left(P_{b}-P\right),
$$

where $\mathbf{n}_{w}$ is the unit outward vector, orthogonal to the vessel wall, $\mathcal{P}$ is the permeability of the vessel, and $P_{b}$ is the oxygen level inside the vessel, which is essentially determined by the haematocrit. Physically, this means that we are assuming that the rate of leakage of oxygen through the walls of the vessels is equal to its rate of diffusion, meaning that the transport process is in steady state. We also impose no-flux boundary conditions along the edges of our domain, $\Omega$ :

$$
\left.\mathbf{n}\right|_{\partial \Omega} \cdot \nabla P=0,
$$

where $\left.\mathbf{n}\right|_{\partial \Omega}$ is the unit outward vector, orthogonal to the boundary of the domain. $P$ is assigned to the state vector as $s_{3}(\mathbf{r})=P(\mathbf{r})$.

Another quantity for which we have to solve a BVP is the distribution of extracellular VEGF. VEGF is secreted by hypoxic cells and released into the extracellular medium when the intracellular concentration reaches a threshold (see sections 5.2 and 6). We make the same adiabatic approximation for VEGF that we have made for oxygen. Hence, the equation we have to solve is

$$
D_{Q} \nabla^{2} Q+k_{Q}(\mathrm{r}) Q=0,
$$

where $Q$ is the extracellular VEGF concentration. In this case, we have a source term given by

$$
k_{Q}(\vec{x})=\left\{\begin{array}{l}
k_{N}^{Q} \text { if there is a normal hypoxic cell at } r \\
k_{C}^{Q} \text { if there is a cancer hypoxic cell at } r \\
0 \text { otherwise }
\end{array}\right.
$$

where $k_{N}^{Q}$ and $k_{C}^{Q}$ are constants. For how we determine when a cell becomes hypoxic we refer the reader to section 6. $Q$ is assigned to the state vector as $s_{4}(\mathbf{r})=Q(\mathbf{r})$.

Details of the numerical procedure used to solve the above BVPs are given in Appendix A of [3]. 


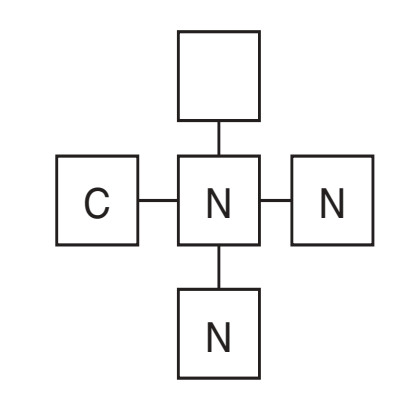

BIGGER THRESHOLD SMALLER THRESHOLD

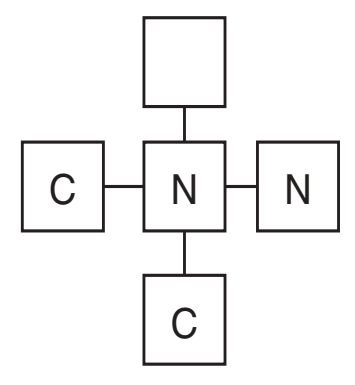

FIG. 7. Two examples showing how thresholds are assigned.

The last issue we deal with here is cell-to-cell interaction. In the present model the only cell-to-cell interaction we consider is competition between the two populations of cells. It is known that under certain circumstances cancer cells modify their environment, providing these cells with a competitive advantage over normal cells, for example by increasing acidity [28, 29]. Tumor cells show greater resistance to acidic environments than their normal counterparts. Consequently, they are able (by using glycolytic metabolism rather than aerobic metabolism) to increase the acidity in their microenvironment, thus providing neighboring cancer cells with a competitive advantage over their normal counterparts. In our model, certain critical decisions (apoptosis, VEGF release, etc.) will be taken when the intracellular concentration of a particular chemical (see section 5) reaches a critical threshold. The way we incorporate such competition is by fixing these thresholds appropriately [3].

The threshold for a cell located at $\mathbf{r}$ to undergo a particular transition (say, for example, apoptosis, which, as detailed in section 5, in our model is controlled by the level of expression of p53) is determined by sampling its neighborhood. If the neighborhood contains more (or the same number of) cells of the same type as the one at $\mathbf{r}$, then the threshold is fixed at a value larger than the value we assign if the neighborhood contains fewer cells of the same type as the one at $\mathbf{r}$. An example is given in Figure 7. Here, in order to decide whether a normal cell will undergo apoptosis, its p53 concentration is compared to a threshold value. If the p53 concentration exceeds the threshold, then the cell will undergo apoptosis. However, to fix the threshold, we consider the neighborhood of the cell under consideration. If, as is the case in the left plot of Figure 7, the neighborhood of our normal cell contains more normal cells than cancer cells, then the threshold value will be fixed to a value higher than that for the situation shown on the right plot of Figure 7, where the neighborhood contains more cancer cells than normal cells. As a result, a normal cell is more resistant to apoptosis when it is surrounded by more normal cells than cancer cells since the amount of p53 that the cell must accumulate in order to undergo apoptosis is larger.

5. Cell division dynamics, apoptosis, and VEGF production. In this section we give details of the internal dynamics of the elements that are occupied by a cell. To those sites, where either $s_{1}(\mathbf{r})=1$ or $s_{1}(\mathbf{r})=2$, we attach a set of ODEs governing cell division, apoptosis, and VEGF production. These ODEs model the evolution of the chemicals controlling those processes at the intracellular level, which determine the "microscopic state" of each element. 
5.1. Cell-cycle: Effects of hypoxia. The dynamics of the cell division cycle can be affected by the extracellular conditions, in particular by the level of extracellular oxygen: low oxygen concentration (hypoxia) yields alterations in the normal progression of the cell through its division cycle [14].

The cell-cycle is the set of events which, eventually, leads to cell division. During the cell-cycle a cell duplicates each of its components, most importantly its chromosomes. The cell-cycle is usually divided into four phases $\left(\mathrm{G}_{1}, \mathrm{~S}, \mathrm{G}_{2}\right.$, and $\left.\mathrm{M}\right)$. The cell goes through two irreversible transitions during its division process. The first of these transitions occurs at the end of $\mathrm{G}_{1}$ : "Start." During $\mathrm{G}_{1}$ the cell monitors its environment and its own size $[6,69]$. When the external conditions and the size of the cell are suitable, the cell commits itself to the process. The second of these transitions, "Finish," 7 occurs when DNA replication is completed. Once the cell has checked that the DNA replication process has taken place without error and that chromatide alignment has been successful, this transition is triggered, and the cell finally undergoes division into two daughter cells.

During $\mathrm{G}_{1}$, activity of cyclin-dependent kinases (CDKs) is low because the relevant cyclin partners are missing: their production is inhibited, and they are rapidly degraded. At "Start" cyclin synthesis is promoted, and hence the CDKs are activated. CDK activity remains high during $\mathrm{S}, \mathrm{G}_{2}$, and $\mathrm{M}$, since it is necessary for DNA replication and other processes occurring during the final stages of the cycle. At "Finish" a protein complex, the anaphase protein complex, is activated and marks specific target proteins (such as cyclins) for degradation by the proteolytic machinery of the cell. This protein complex is composed of a dozen polypeptides and two auxiliary proteins: Cdc20 and Cdh1. When active, these two proteins present the target proteins to the core of the complex for labeling. Together, they label cyclins for destruction at the end of the cycle, allowing the control system to return to $\mathrm{G}_{1}$. Cdc20 and Cdh1 activity is also controlled by cyclin-CDK complexes. However, cyclin-CDKs control each protein differently: while cyclin-CDK activates Cdc20, it inhibits Cdh1.

Despite the great number of differences between the cell-cycle of cancerous and normal cells [25], some mechanisms are common to both types of cell. In particular, regulation of the transition through the check points is accomplished by the CDK network in both cases.

In normal cells hypoxia simply slows down (arrests) the division process, whereas in cancer cells it stimulates both arrest and quiescence; i.e., cells go into a latent state in which most of their functions, including proliferation, are suspended. The ability of cancer cells to go into a quiescent state provides them with a remarkable resistance to hypoxia [64].

Building on a previous model [69] and experimental information on the effects of hypoxia on the cell-cycle [27], we have formulated a model which accounts for arrest of the transition through the restriction point under hypoxic stress and (some of) the differences in the response to hypoxia of cancer and normal cells (see [4]). To model the effect of hypoxia on the cell-cycle we have to consider a new element in the CDK network $[25,27]$, the protein $\mathrm{p} 27$, which inhibits the activity of the complex Cyc-CDK, thus inhibiting DNA synthesis. It has been shown that $\mathrm{p} 27$ production is stimulated by hypoxia, thus mediating hypoxia-induced arrest of the $\mathrm{G}_{1} / \mathrm{S}$ transition. In [27] the authors were able to gather experimental evidence to construct the picture of hypoxiainduced $\mathrm{G}_{1}$ arrest: hypoxia causes an overexpression of $\mathrm{p} 27$, which downregulates the activity of the cyclin-CDK complexes, which, in turn, prevents normal progress of the

\footnotetext{
${ }^{7}$ We have adopted the nomenclature used by [69].
} 
cell through the "Start" transition (see [27] and [4] for a complete account). Note that the model that Tyson and Novak presented in [69] was originally formulated for yeast. However, yeast has been extensively used as an experimental model system for the study of the cell-cycle in eukariotic cells, since the basic regulatory mechanisms are believed to be the same for all eukariotic cells, including mammalian cells [6]. Thus, we have used the model reported in [69], although the actual CDK regulatory network is more complex in mammalian cells. The experiments reported in [27] on the effects of hypoxia on the cell-cycle were carried out using fibroblasts.

Based on these observations, the model that we propose (full details given in [4]) is

$$
\begin{aligned}
& \frac{d x(\mathbf{r})}{d t}=\frac{\left(k_{3}^{\prime}+k_{3}^{\prime \prime} u(\mathbf{r})\right)(1-x(\mathbf{r}))}{J_{3}+1-x(\mathbf{r})}-\frac{k_{4} m(\mathbf{r}) y(\mathbf{r}) x(\mathbf{r})}{J_{4}+x(\mathbf{r})}, \\
& \frac{d y(\mathbf{r})}{d t}=k_{1}-\left(k_{2}^{\prime}+k_{2}^{\prime \prime} x(\mathbf{r})+k_{2}^{\prime \prime \prime} z(\mathbf{r})\right) y(\mathbf{r}), \\
& \frac{d m(\mathbf{r})}{d t}=\mu\left(\frac{y(\mathbf{r})}{y(\mathbf{r})+y_{0}}\right) m(\mathbf{r})\left(1-\frac{m(\mathbf{r})}{m_{*}}\right), \\
& \frac{d z(\mathbf{r})}{d t}=\chi(m, \mathbf{r})-k_{5}^{\prime} \frac{P(\mathbf{r})}{B+P(\mathbf{r})} z(\mathbf{r}), \\
& \frac{d u(\mathbf{r})}{d t}=k_{6}^{\prime}-\left(k_{6}^{\prime}+k_{6} y(\mathbf{r})\right) u(\mathbf{r}), \\
& v=1-u,
\end{aligned}
$$

where $x$ is the concentration of Cdh1, an antagonist of cyc-CDK, $y$ is the concentration of cyc-CDK complexes, $m$ is the mass of the cell, $z$ is the concentration of p27, $u$ is the concentration of nonphosphorylated (RB), and $v$ is the concentration of phosphorylated RB (RBP). The dependence on $\mathbf{r}$ reminds us that this is a local, internal dynamics.

In (5.4) the production rate for $\mathrm{p} 27, \chi(m, \mathbf{r})$, depends on the type of cell at $\mathbf{r}$ :

$$
\chi(m, \mathbf{r})=\left\{\begin{array}{l}
\left.k_{5} \text { if } \mathrm{s}_{1}(\mathbf{r})=1 \text { (i.e., if there is a cancer cell at } \mathbf{r}\right), \\
\left.k_{5}\left(1-\frac{m(\mathbf{r})}{m_{*}}\right) \text { if } \mathrm{s}_{1}(\mathbf{r})=2 \text { (i.e., if there is a normal cell at } \mathbf{r}\right), \\
0 \text { otherwise. }
\end{array}\right.
$$

We have assumed that p27 production is modulated by cell growth in normal cells, whereas no such control has been assumed to act on p27 production in cancer cells. We refer the reader to [4] for a detailed discussion of the experimental evidence supporting this assumption.

We have introduced here a new element with respect to the model presented in [4], namely, a dependence of the growth rate of the mass of the cell on the concentration of cyc-CDK complexes (see (5.3)). In general, cell growth is not independent of the external conditions nor of its own state of development. The dependence of $m$ on $y$ in (5.3) is intended to account for this. For example, if the level of oxygen is low, the cell will arrest in $\mathrm{G}_{1}$, and the concentration of cyc-CDK complexes will be kept low longer. This, according to (5.3), will slow down cell growth and is consistent with the fact that low levels of oxygen yield a smaller growth rate. However, this modification changes neither the basic results obtained in [4] nor their mechanisms.

In addition, if $\mathbf{s}(\mathbf{r})=1,2$, we define a phase, $\phi(\mathbf{r})$, which gives the position of the 
(a)

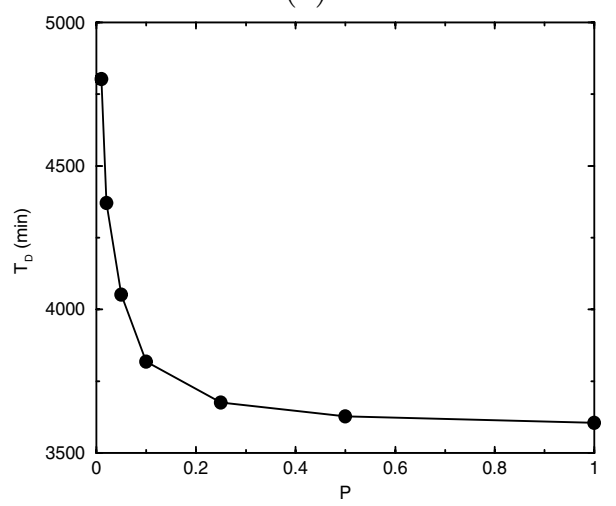

(b)

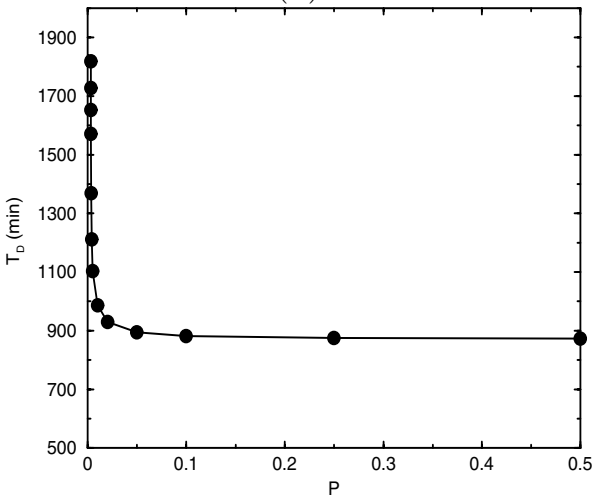

FIG. 8. Division time as a function of the oxygen tension, $P$. (a) normal cells; (b) cancer cells. Note the vertical asymptote in (b) at $P \simeq 0.003$, which is the critical value of the oxygen tension for the transition to hypoxia in cancer cells. Note that in [20] doubling times for V-79 (cancer) cells are reported as 10-19 hours (600-1140 minutes) which are in fairly good agreement with the division times we obtain from our model.

cell in the cell-cycle. Its dynamics is given by

$$
\frac{d \phi(\mathbf{r})}{d t}=\eta(x(\mathbf{r}), y(\mathbf{r}))-\sin \left(\phi(\mathbf{r})-\phi_{0}\right)
$$

where

$$
\eta(x(\mathbf{r}), y(\mathbf{r}))=\left\{\begin{array}{l}
0 \text { if } y(\mathbf{r})<T H R_{1}, \\
0.5 \text { if } y(\mathbf{r})>T H R_{1} \text { and } y(\mathbf{r})<T H R_{2}, \\
1.5 \text { if } y(\mathbf{r})>T H R_{2} \text { and } x(\mathbf{r})<T H R_{3}
\end{array}\right.
$$

with $T H R_{1}<T H R_{2}$. Equation (5.8) is such that when $\eta(x(\mathbf{r}), y(\mathbf{r}))=0$ there is a stable fixed point $\phi_{\infty}=\phi_{0}$. Since $\eta=0$ when $y(\mathbf{r})$ is very low, this fixed point corresponds to the cell being in $\mathrm{G}_{1}$. If $0<\eta(x(\mathbf{r}), y(\mathbf{r}))<1$, then (5.8) has an unstable fixed point and a stable fixed point $\phi_{\infty}>\phi_{0}$. Since this is the situation when $y(\mathbf{r})$ is bigger than some threshold, this fixed point corresponds to a cell in $\mathrm{S}_{-} \mathrm{G}_{2}-\mathrm{M}$. If $\eta(x(\mathbf{r}), y(\mathbf{r}))>1$, there are no fixed points of (5.8), which means that the cell has gone through all the check points and is committed to finishing the cell-cycle. When $\phi(\mathbf{r})=2 \pi$ the cell divides and the control systems of both the new and old cells are (re)set to the initial state: $x(\mathbf{r})=0.9, y(\mathbf{r})=0.001, m(\mathbf{r})=5, z(\mathbf{r})=0, u(\mathbf{r})=0$, $\phi(\mathbf{r})=0$.

We can count the number of divisions a given cell has undergone, $n(\mathbf{r})$, using $\phi(\mathbf{r})$ :

$$
n(\mathbf{r})=\int_{0}^{\infty} \delta_{\phi(\mathbf{r}, t), 2 \pi} d t .
$$

In our lattice element, $n$ is assigned to the state vector as $s_{2}(\mathbf{r})=n(\mathbf{r})$, and the substances controlling the cell-cycle are stored as $s_{5}(\mathbf{r})=x(\mathbf{r}), s_{6}(\mathbf{r})=y(\mathbf{r})$, $s_{7}(\mathbf{r})=m(\mathbf{r}), s_{8}(\mathbf{r})=z(\mathbf{r}), s_{9}(\mathbf{r})=u(\mathbf{r}), s_{10}(\mathbf{r})=v(\mathbf{r})$, and $s_{11}(\mathbf{r})=\phi(\mathbf{r})$.

We now summarize the most significant results of our cell-cycle models of normal and cancer cells (see [4] for a full analysis). Both models produce hypoxia-induced arrest of the $\mathrm{G}_{1} / \mathrm{S}$ transition, which yields longer division times (see Figure 8). However, 
if we look at Figure 8(b), where we have shown how the division times for cancer cells vary with the oxygen tension, $P$, we observe a vertical asymptote for a given value of the oxygen tension. Such behavior is not observed in normal cells (see Figure 8(a)). The origin of this behavior is that the cancer cell model exhibits (hypoxia-induced) quiescence, i.e., the ability of halting the cell-cycle, rather than merely delaying or arresting it. Such a behavior is actually observed in cancer cells [4]. In fact, the ability of cancer cells to enter a quiescent state under hypoxic stress is one of the main reasons why cancer cells are so remarkably resistant to hypoxia [64]. There is experimental evidence that quiescent cells are much less susceptible to apoptosis than proliferating cells $[18,49]$. On the other hand, since transition to quiescence is reversible, cancer cells might wait in the quiescence state (where they are "protected" from apoptosis) while normal cells are starving and dying. This allows them to wait until the total (normal+cancer) cell density has diminished and there is more oxygen available, so they can come back to the proliferative state.

Another feature that emerges from our cancer cell model concerns the behavior of the concentration of p27 averaged over a period of division:

$$
\langle z\rangle=\frac{1}{T_{D}} \int_{0}^{T_{D}} z(t) d t
$$

and its dependence on $k_{5}$ and $k_{5}^{\prime}$ regulate the production and decay rates of p27 (see (5.4)). This quantity increases as the ratio $k_{5} / k_{5}^{\prime}$ increases. On the other hand, the division time also increases with $k_{5} / k_{5}^{\prime}$. From this result we can predict that there is a correlation between the proliferation rate of the cancer cells and $\langle z\rangle[4]$ : the latter decreases when the former increases. This agrees with a recent clinical trial in which low levels of p27 appear to be a poor prognostic factor [43]. We will return to this in section 7.2 .

5.2. Other responses to hypoxia: Apoptosis and VEGF production. In this section we account for the intracellular dynamics of the other two hypoxiainduced cellular responses that we consider, namely, apoptosis and VEGF production. We formulate models for the levels of expression of the tumor suppressor gene p53 and the intracellular concentration of VEGF.

The p53 gene plays a fundamental role in many biological processes, including tumor growth $[64,26]$ and hypoxia-induced cellular responses. In particular, the wild-type p53 gene mediates apoptosis (programmed cell death) in various situations, such as DNA damage, hypoxia, the presence of certain cytokines, and metabolic changes [42]. It has been shown in several studies [64,31,65] that cells which have lost p53 expression (null cells) or have acquired a mutation in the p53 gene (mutant cells) can survive under hypoxia for longer periods than their wild-type counterparts due to the decrease in apoptosis caused by abnormal functioning of the p53 gene. Moreover, it has been shown that tumor cells expressing mutant p53 have an adaptive advantage over tumor cells expressing wild-type p53 [26].

In addition to cell-cycle arrest, hypoxia induces other cellular responses (see Figure 9). As shown in Figure 9(a), in both normal and cancer cells, hypoxia stimulates VEGF production, p53 expression, and apoptosis. In normal cells, p53 expression stimulates apoptosis and may also inhibit VEGF production [64]. In many cases, mutations in p53 in cancer cells may lead to a scenario in which p53 apparently upregulates VEGF production and stops triggering apoptosis [64]. This last scenario is the one we consider in our model. Figure 9(b) shows the corresponding hypoxiainduced response in (mutant) cancer cells. In this case, hypoxia also induces VEGF 
(a)

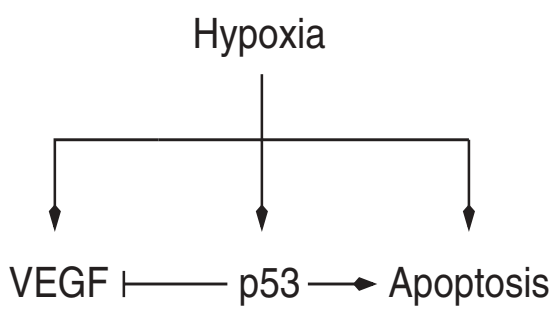

(b)

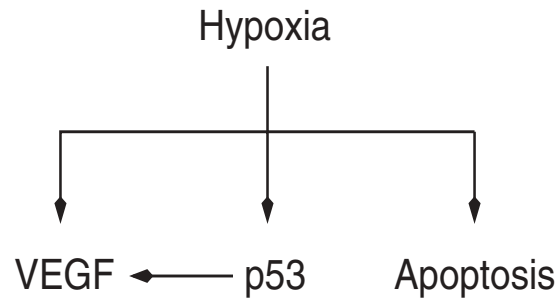

FIG. 9. In addition to cell-cycle arrest, hypoxia induces other cellular responses, which are schematically shown in this diagram for (a) normal cells and (b) cancer cells. See [64].

production, p53 expression, and apoptosis. However, the role of the p53 gene is different: we assume that p53 does not seem to alter the rate of hypoxia-induced apoptosis and, in contrast to what happens in normal cells, it inhibits VEGF production.

The model we propose to account for levels of expression of p53 is the same for normal and cancer cells. We assume that the background production rate is constant and that the decay rate is modulated by the local level of extracellular oxygen:

$$
\frac{d p(\mathbf{r})}{d t}=k_{7}-k_{7}^{\prime} \frac{P(\mathbf{r})}{C+P(\mathbf{r})} p(\mathbf{r})
$$

where $p$ represents the concentration of $\mathrm{p} 53$ within a given cell.

When considering intracellular VEGF, we distinguish between normal and cancer cells:

$$
\frac{d q(\mathbf{r})}{d t}=\xi(p, q, \mathbf{r})-k_{8}^{\prime} \frac{P(\mathbf{r})}{D+P(\mathbf{r})} q(\mathbf{r}),
$$

where $q$ represents the concentration of VEGF and

$$
\xi(p, q, \mathbf{r})=\left\{\begin{array}{l}
k_{8}+k_{8}^{\prime \prime} \frac{p q}{J_{5}+q} \text { if } \mathbf{s}_{1}(\mathbf{r})=1(\text { cancer cell }) \\
k_{8}-k_{8}^{\prime \prime} \frac{p q}{J_{5}+q} \text { if } \mathbf{s}_{1}(\mathbf{r})=2(\text { normal cell }) .
\end{array}\right.
$$

In (5.13) the dependence on the extracellular level of oxygen accounts for the fact that VEGF expression is stimulated by hypoxia. Equation (5.14) accounts for the different regulation of p53 expression in normal and cancer cells (see Figure 9). We have assumed Michaelis-Menten kinetics for the inhibition (activation) of VEGF by p53 in normal (cancer) cells.

The intracellular concentration of VEGF is assigned to the automaton state vector as $s_{12}(\mathbf{r})=q(\mathbf{r})$ and the level of expression of $\mathrm{p} 53$ as $s_{13}(\mathbf{r})=p(\mathbf{r})$.

To solve numerically the systems of ODEs we use a four-stage Runge-Kutta method [56] with an extended stability region [70].

6. Rules for the evolution of our physiologically structured model. The rules governing the evolution of the automaton elements are as follows.

1. An element that is empty or occupied by a vessel does not evolve directly. However, an empty element can change its state (to an occupied element) when cell division takes place in a neighboring element that is occupied by a cell. 
2. The distribution of oxygen is calculated by solving an appropriate boundary value problem, as described in section 4 .

3. We determine the type of cell in an occupied element from the first component of its state vector. At each time step we solve the ODEs determining the internal (cell-cycle, VEGF production, and p53 expression) dynamics of the cell. The internal dynamics are modulated by the extracellular oxygen, as explained in section 5 .

4. Cells (either normal or cancerous) attempt division when the local phase $\phi(\mathbf{r})=2 \pi$.

5. When a cell attempts division, we sample in a region of radius $R$ around the element. ${ }^{8}$ If there is only one empty space, then the cell divides, and the new cell occupies this empty space. If there is more than one empty space, the new cell goes to the free element with the largest oxygen concentration [52]. If there is no empty space, the cell fails to divide and dies [40].

6. When the oxygen concentration is low, normal cells become sources of VEGF. This process is controlled in turn by the local intracellular concentration of VEGF (which is controlled by the concentration of extracellular oxygen and the level of expression of p53 (section 5.2)). If $q(\mathbf{r})$ is above a threshold value, $q_{T H R}$, the cell releases VEGF into the extracellular medium. We determine the threshold value by sampling the occupation state of its nearest neighbors, as explained in section 4 .

7. Under sustained hypoxia, normal cells die. This process is controlled by the local level of p53 (which is controlled by the concentration of extracellular oxygen (section 5.2)). If $p(\mathbf{r})$ is above a threshold value, $p_{T H R}$, the cell dies. We determine the threshold value by sampling the occupation state of its nearest neighbors, as explained in section 4 .

8. Cancer cells whose local oxygen concentration is very low enter a quiescent or latent state, during which most of the cell functions are suspended, including proliferation (section 5.1). On entering this state, a clock is started. The clock is incremented by one unit for each iteration the cell remains in the quiescent state, i.e., while the oxygen level remains below threshold. If the clock reaches a given value the cell dies. However, if at some time the oxygen level goes above threshold the cell returns to the proliferating state and its clock is reset to zero. Cancer cells in the quiescent state become sources of VEGF. The transition from the "proliferative" to the quiescent state is triggered by the local concentration of $\mathrm{p} 27$ : when $z(\mathbf{r})$ is above a threshold, $z_{T H R}$, the cell enters the quiescent state. In this case the threshold is fixed by the internal dynamics, and no sampling of the environment is done.

9. Elements occupied by normal and cancer cells are sinks of oxygen.

10. Extracellular VEGF diffuses through the tissue according to the BVP formulated in section 4. VEGF eventually reaches the vasculature and interacts with it through the vascular adaptation mechanism, (3.3). This changes the structure of the network and therefore the distribution of haematocrit (section 3), thereby yielding a new oxygen distribution.

The internal state of each one of the (occupied) elements of the lattice is updated synchronously. These rules are summarized in the flow chart in Figure 10.

The values of parameters used in our simulations are shown in Tables 1 and 2 .

\footnotetext{
${ }^{8}$ In the simulations below, $R=1$ in element units.
} 


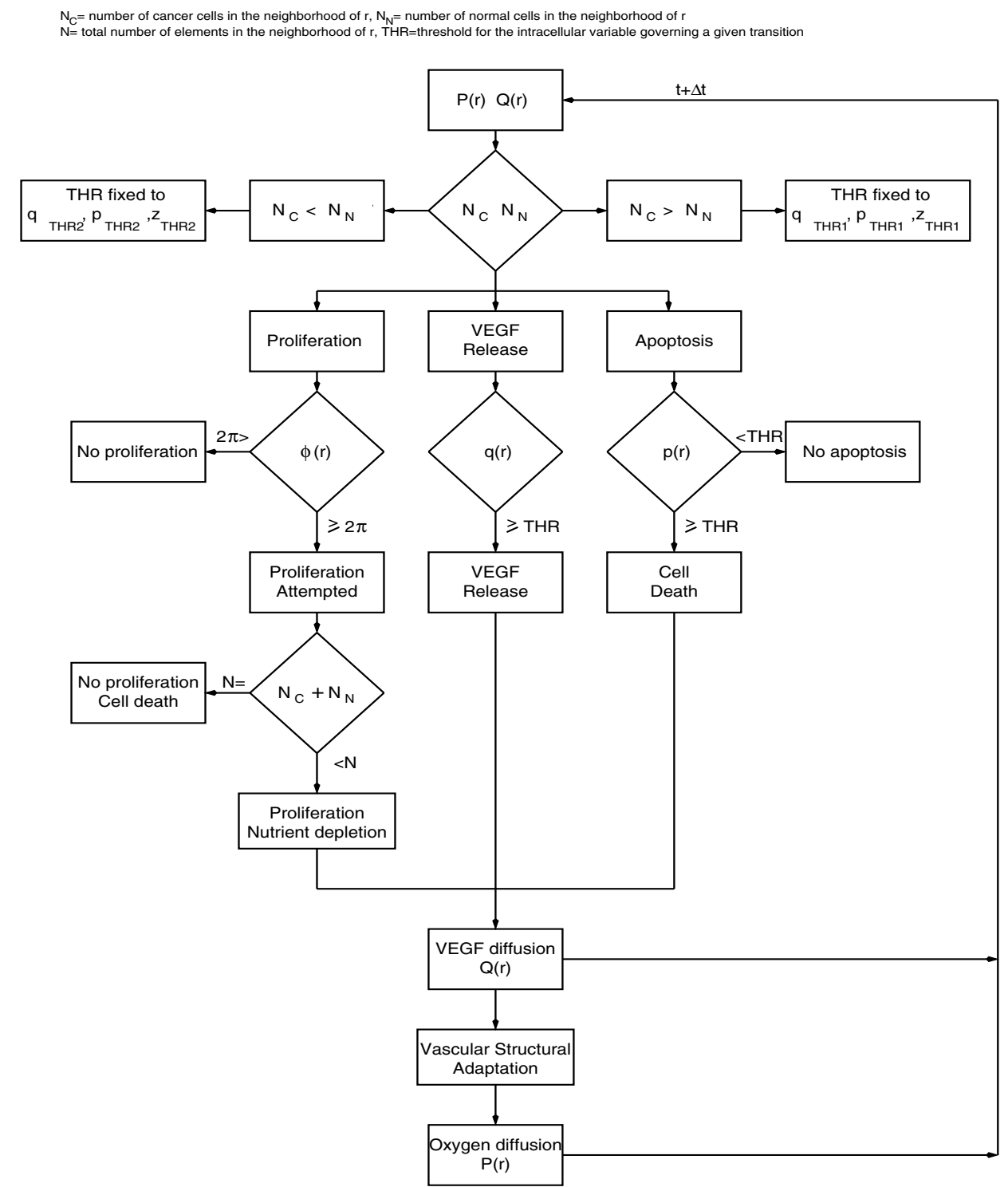

FIG. 10. Flow chart corresponding to the computer algorithm we have used to implement the rules presented in section 6.

\section{Patterns of growth and invasion: Implications for therapy.}

7.1. Growth and invasion. As in [3], we have performed two types of simulated experiments: the growth of a colony (either a two- or one-species colony) occupying empty space and the invasion of a healthy tissue by a malignant colony.

In this section we use our model to study how growth and invasion occur in three different situations, namely, in a homogeneous environment (uniform distribution of oxygen), in an inhomogeneous environment with vasculature decoupled from the tissue, and, finally, in the most complex situation in which the vasculature and tissue are coupled. To see the differences between the patterns of growth in the different 
TABLE 1

Parameter values used for our numerical computations. These values have been used to produce all the results shown in this paper apart from in Figure 17. For these, we have used $k_{5}^{\prime}=0.01$ and the value of $k_{5}$ prescribed by the corresponding value of $k_{5} / k_{5}^{\prime}$. The "estimated" values have been calculated to fit the values of the duplication times of V-79 cancer cells reported by [20]. The parameters for which we do not have estimates (marked as "see caption" in the table) have been fixed according to biologically sensible criteria. The parameter values involved in p53 regulation have been fixed in such a way that normal cells undergo apoptosis when cancer cell enter quiescence [64]. The parameter values involved in VEGF expression have been fixed in order to have VEGF secretion before p53-induced apoptosis.

\begin{tabular}{|c|c|c|c|c|}
\hline Parameter & Value (normal) & Value (cancer) & Units & Source \\
\hline \hline$k_{1}$ & 0.04 & 0.04 & $\min ^{-1}$ & {$[69]$} \\
\hline$k_{2}^{\prime}$ & 0.5 & 0.4 & $\min ^{-1}$ & estimated \\
\hline$k_{2}^{\prime \prime}$ & 1 & 1 & $\min ^{-1}$ & {$[69]$} \\
\hline$k_{2}^{\prime \prime \prime}$ & 0.25 & 0.25 & $\min ^{-1}$ & estimated \\
\hline$k_{3}^{\prime}$ & 1 & 1 & $\min ^{-1}$ & {$[69]$} \\
\hline$k_{3}^{\prime \prime}$ & 10 & 10 & $\min ^{-1}$ & {$[69]$} \\
\hline$k_{4}^{\prime}$ & 35 & 35 & $\min ^{-1}$ & {$[69]$} \\
\hline$\mu$ & 0.01 & 0.01 & $\min ^{-1}$ & {$[69]$} \\
\hline$m_{*}$ & 10 & 10 & none & {$[69]$} \\
\hline$J_{3}, J_{4}$ & 0.04 & 0.04 & none & {$[69]$} \\
\hline$k_{5}$ & 0.1 & 0.002 & $\min ^{-1}$ & estimated \\
\hline$k_{5}^{\prime}$ & 0.01 & 0.01 & $\min ^{-1}$ & estimated \\
\hline$B$ & 0.01 & 0.01 & none & estimated \\
\hline$k_{6}$ & 0.01 & 0.01 & $\min ^{-1}$ & estimated \\
\hline$k_{6}^{\prime}$ & 0.1 & 0.1 & $\min ^{-1}$ & estimated \\
\hline$k_{7}$ & 0.002 & 0.002 & $\min ^{-1}$ & see caption \\
\hline$k_{7}^{\prime}$ & 0.01 & 0.01 & $\min ^{-1}$ & see caption \\
\hline$C$ & 0.01 & 0.01 & none & see caption \\
\hline$k_{8}$ & 0.002 & 0.002 & $\min ^{-1}$ & see caption \\
\hline$k_{8}^{\prime}$ & 0.01 & 0.01 & $\min ^{-1}$ & see caption \\
\hline$k_{8}^{\prime \prime}$ & 0.002 & 0.002 & $\min ^{-1}$ & see caption \\
\hline$D$ & 0.01 & 0.01 & none & see caption \\
\hline$J_{5}$ & 0.04 & 0.04 & none & see caption \\
\hline
\end{tabular}

cases, we focus on a simple case: a one-species colony composed only of normal cells. However, the results for cancer cells or mixed populations are not significantly different.

In Figure 11, we have plotted the size of such a colony as a function of time under the three conditions we have mentioned. We observe (Figure 11(a)) that in the case of a homogeneous environment, as in [3], the colony grows in a regular fashion until it occupies all the space available. In fact, Figure 12 shows how in this case the colony grows in a symmetric way. By contrast, when the same colony is grown under an inhomogeneous distribution of haematocrit (see Figure 4), the colony grows to a saturation size (Figure 11(b)) and follows a pattern of spreading that is much more heterogeneous (Figures 12(d), (e), and (f)). A similar phenomenon is observed when we couple vascular structural adaptation and colony development: the colony does not take over all the space available, and the pattern of spreading is rather heterogeneous (see Figure 11(c) and Figures 12(g), (h), and (i)). However, one difference is observed: the colony grows to a significantly larger saturation size. These results are consistent 
TABLE 2

Parameter values used in our simulations. The values of the $z_{T H R 1}$ and $z_{T H R 2}$ are fixed by the cell-cycle model. They correspond to the critical stationary value of $p 27$ such that the cancer cells enter quiescence. The parameters that we have not been able to estimate, marked as "see caption" in the table, have been fixed in order to meet biologically sensible requirements. We have also performed sensitivity analysis with respect to these parameters. Our results are robust to changes in these parameters. $p_{T H R 2}$ is actually fixed by $z_{T H R 2}$ (see the caption of Table 1 ).

\begin{tabular}{|c|c|c|c|}
\hline Parameter & Value & Units & Source \\
\hline \hline$k_{m}$ & 0.83 & $s^{-1}$ & {$[60]$} \\
\hline$Q_{r e f}$ & 40 & $\mathrm{nl} / \mathrm{min}$ & {$[60]$} \\
\hline$k_{s}$ & 1.79 & $s^{-1}$ & {$[60]$} \\
\hline$\alpha$ & 0.5 & none & {$[24]$} \\
\hline THR & 2.5 & none & {$[24]$} \\
\hline$D_{P}$ & $2.41 \cdot 10^{-5}$ & $\mathrm{~cm}^{2} s^{-1}$ & {$[30]$} \\
\hline$K_{N}$ & $1.57 \cdot 10^{-4}$ & $\mathrm{mlO}_{2} \mathrm{ml}^{-1} s^{-1}$ & {$[30]$} \\
\hline$K_{T}$ & $1.57 \cdot 10^{-4}$ & $\mathrm{mlO}_{2} \mathrm{ml}^{-1} \mathrm{~s}^{-1}$ & estimated \\
\hline $\mathcal{P}$ & $3.0 \cdot 10^{-4}$ & $\mathrm{~cm} \mathrm{~s}^{-1}$ & estimated \\
\hline$q_{T H R 1}$ (normal cells only) & 1.5 & none & see caption \\
\hline$q_{T H R 2}$ (normal cells only) & 2 & none & see caption \\
\hline$p_{T H R 1}$ (normal cells only) & 0.08 & none & see caption \\
\hline$p_{T H R 2}$ (normal cells only) & 0.8 & none & see caption \\
\hline$z_{T H R 1}$ (cancer cells only) & 0.8 & none & estimated \\
\hline$z_{T H R 2}$ (cancer cells only) & 0.8 & none & estimated \\
\hline
\end{tabular}

(a)

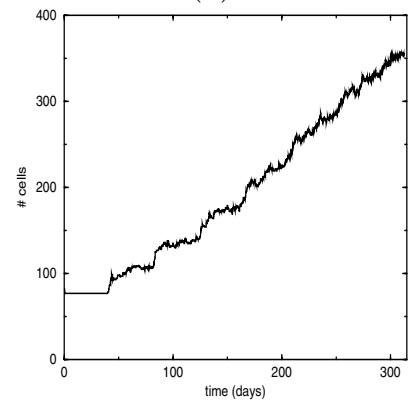

(b)

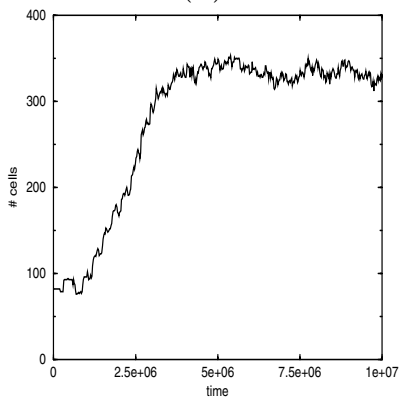

(c)

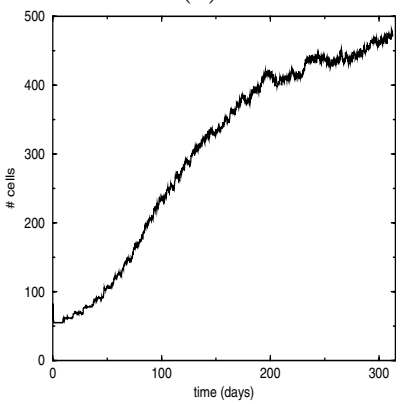

FIG. 11. (a) Growth of a colony of normal cells under a homogeneous distribution of haematocrit in the vasculature. (b) Growth of a colony of normal cells under an homogeneous distribution of haematocrit, with vasculature decoupled from tissue. (c) Growth of a colony of normal coupling between tissue and vasculature. In (a) and (c) the steady state is not shown because the of the computing time required.

with the ones we obtained from our previous model [3].

Now, we consider the case of a two-species (normal and cancer) cell population. Two scenarios will be considered: colony growth and invasion. In the first scenario, the colony is initially composed of roughly 50 percent of each type of cell. In the second scenario, invasion, an initial colony of cancer cells is placed in a "tissue" composed of normal cells, empty spaces, and vasculature. The aim is to determine conditions under which cancer cells can take over the tissue. In both cases we assume that the vasculature and tissue are coupled.

The results for a two-species colony are shown in Figures 13 and 14. We find that, 
(a)

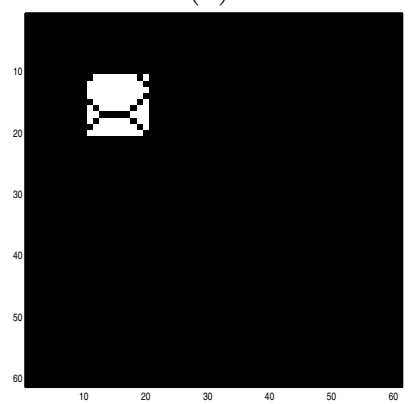

(d)

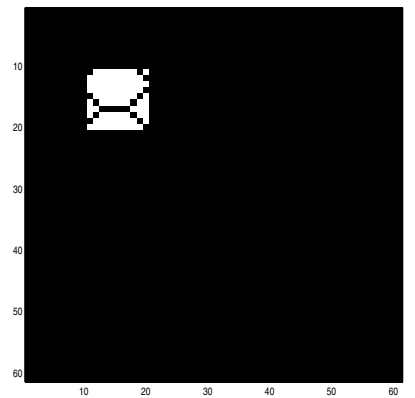

$(\mathrm{g})$

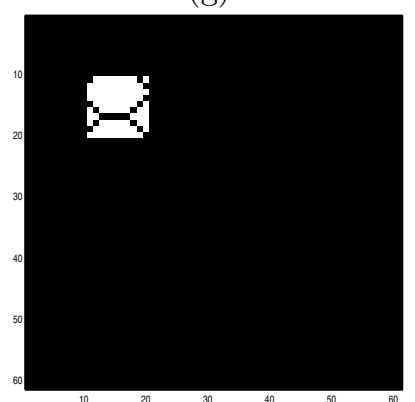

(b)

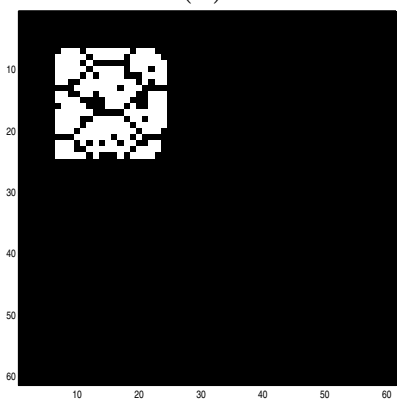

(e)

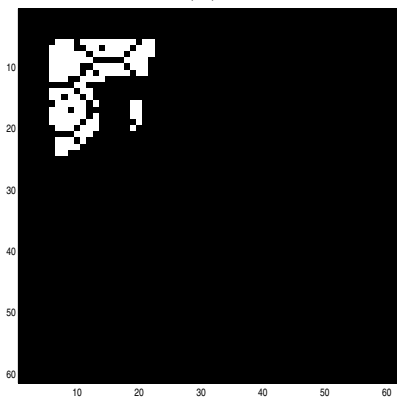

(h)

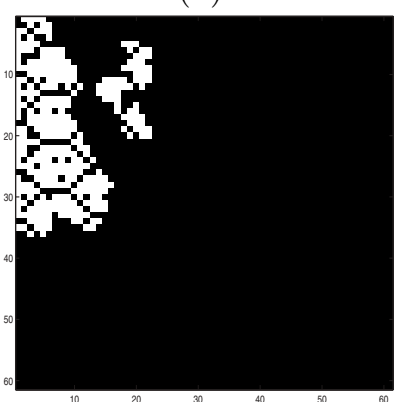

(c)

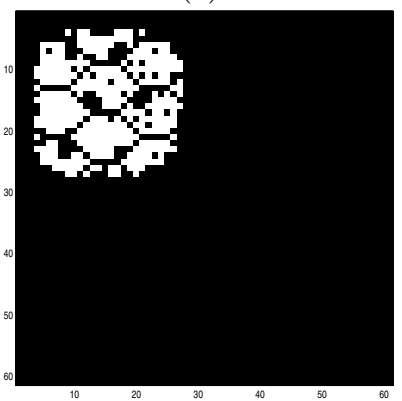

(f)

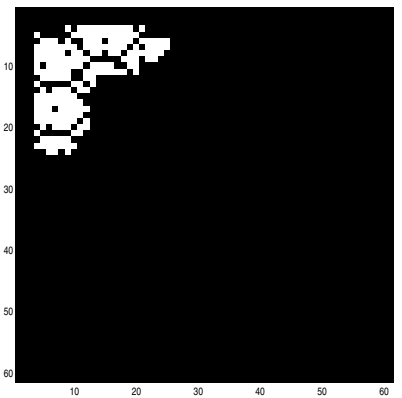

(i)

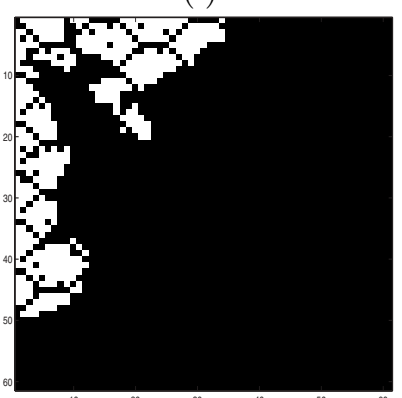

FIG. 12. In (a), (b), and (c) we show three snapshots showing the spatial distribution of cells for a colony of normal cells growing under a homogeneous distribution of haematocrit (see Figure 11(a)). In (a) we have plotted the initial condition, (b) corresponds to the colony after 150 days, and (c) corresponds to 310 days. In (d), (e), and (f) we show three snapshots showing the spatial distribution of cells for a colony of normal cells growing under inhomogeneous distribution of haematocrit, with vasculature decoupled from tissue (see Figure 11(b)). In (a) we have plotted the initial condition, (b) corresponds to the colony after 75 days, and (c) corresponds to 150 days. In $(\mathrm{g}),(\mathrm{h})$, and (i) we show three snapshots showing the spatial distribution of cells for a colony of normal cells growing under inhomogeneous distribution of haematocrit, with vasculature-tissue coupling (see Figure 11(c)). In (a) we have plotted the initial condition, (b) corresponds to the colony after 150 days, and (c) corresponds to 310 days.

for the parameter values shown in Tables 1 and 2, our model predicts that cancer cells rapidly dominate the normal cells. Therefore, the cancer cells are better competitors than normal cells.

The results shown in Figures 15 and 16 correspond to invasion of a healthy tissue by a malignant colony. Due to the better competitive character of the cancer cells, the initial small colony (Figure 16(a)) spreads over a significant part of the tissue 
(a)

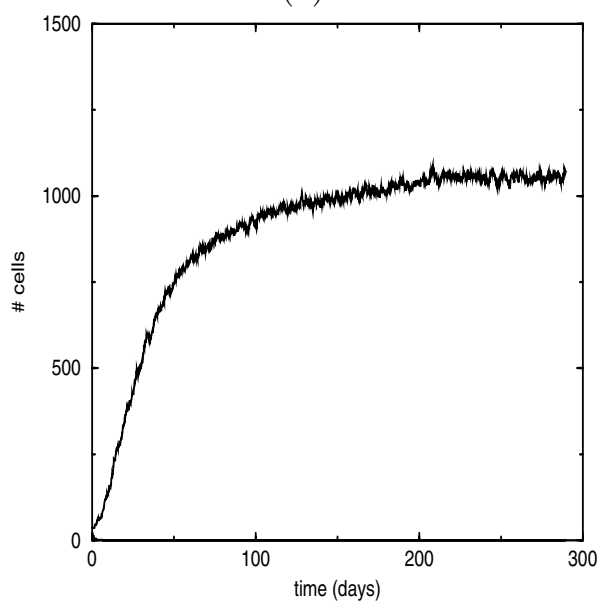

(b)

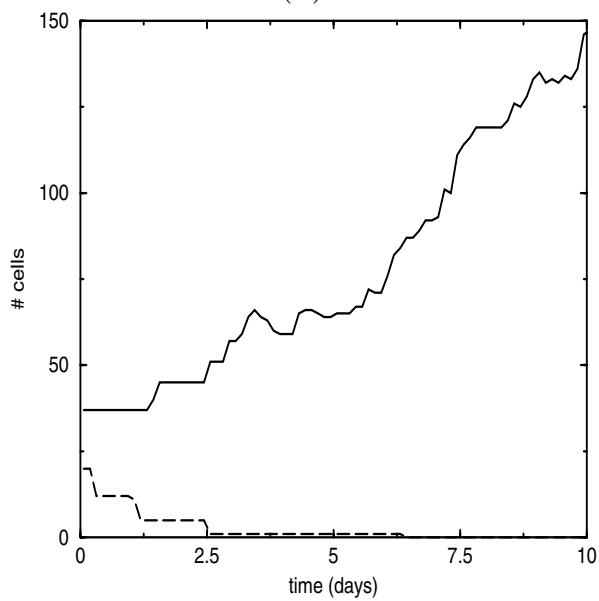

FIG. 13. (a) Time evolution of the size of the colony for $k_{5} / k_{5}^{\prime}=0.2$. (b) A close-up of the initial stages. The dashed line corresponds to normal cells, whereas the solid line corresponds to cancer cells.

TABLE 3

Spatial average of 27 protein expressed by cancer cells for three different values of $k_{5} / k_{5}^{\prime}$, as calculated from the patterns shown in Figures 17(d), (e), and (f).

\begin{tabular}{|c|c|}
\hline$k_{5} / k_{5}^{\prime}$ & $\bar{z}$ \\
\hline \hline 0.2 & 0.36 \\
\hline 0.6 & 0.67 \\
\hline 0.7 & 0.83 \\
\hline
\end{tabular}

initially occupied by normal cells. In the regions invaded by the cancer cells, normal cells have been completely eliminated.

7.2. Implications for therapy. As mentioned in section 5.1, a recent clinical trial has concluded that low levels of p27 in high-grade astrocytomas constitute a poor prognostic indicator [43]. The authors of this study attribute this feature to two factors: increased proliferative activity and decreased apoptosis produced by low levels of $\mathrm{p} 27$.

In section 5.1, we have shown that our model of the cell-cycle reproduces the first of these characteristics: low levels of p27 correlate with smaller division times [4]. In our model this is achieved by changing the ratio $k_{5} / k_{5}^{\prime}$ : when this ratio increases, both the concentration of p27 and the division time increase.

We have carried out model simulations introducing into the cell-cycle model for the cancer cells different values of the ratio $k_{5} / k_{5}^{\prime}$. We have measured the quantity:

$$
\bar{z}=\frac{1}{N_{c}} \sum_{\mathbf{r} \in I} z(\mathbf{r}) \delta_{\mathbf{s}_{1}(\mathbf{r}), 1},
$$

which is the spatial average of p27 protein expressed by the cancer cells. In (7.1) $N_{c}$ is the total number of cancer cells. Our results show that $\bar{z}$ increases with $k_{5} / k_{5}^{\prime}$ (see Table 3).

On the other hand, if we look at the evolution of the colonies of normal and 
(a)

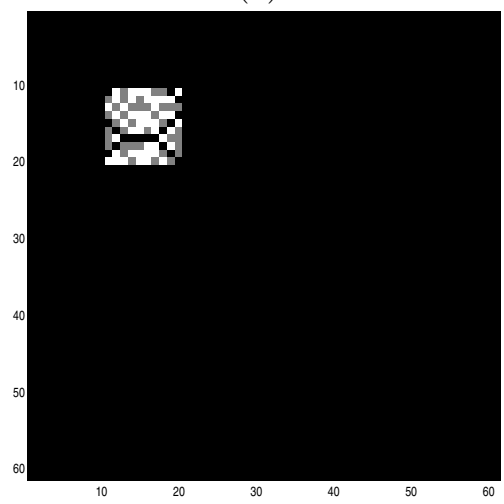

(c)

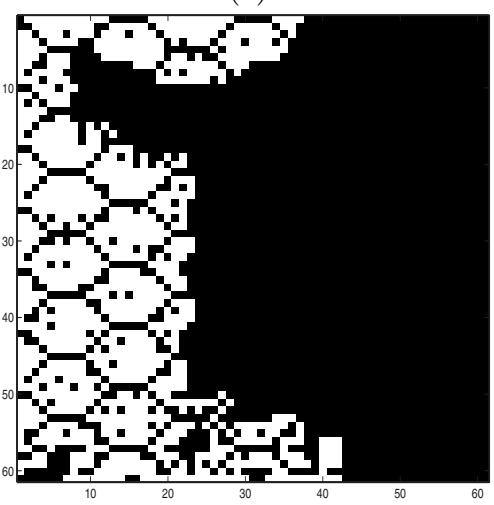

(b)

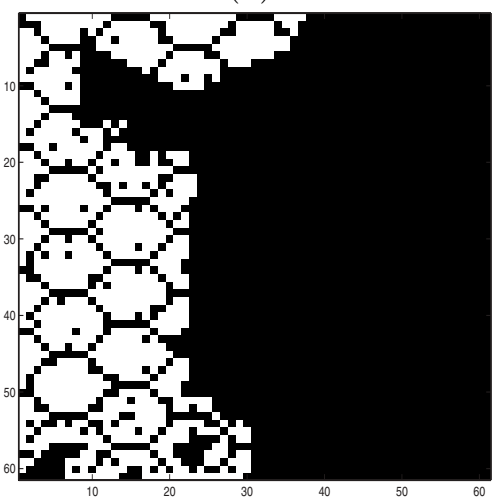

(d)

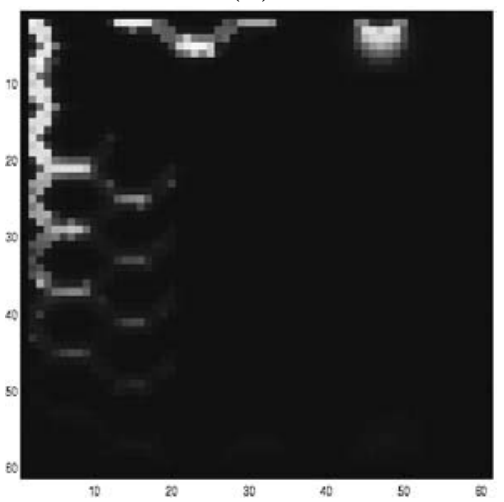

FIG. 14. (a), (b), and (c) show three snapshots of the spatial distribution of cells for a colony of normal and cancer cells, corresponding to 0, 100, and 310 days, respectively. White elements represent cancer cells, whereas grey elements correspond to normal cells. Black elements are either empty or occupied by vessels. (d) shows the distribution of oxygen when the colony has attained its stationary state. In (d) the greyscale corresponds to the concentration of oxygen: lighter grey corresponds to higher oxygen levels.

cancer cells (see Figures 17(a), (b), and (c)), we observe that for $k_{5} / k_{5}^{\prime}=0.2$ (Figure $17(\mathrm{a}))$ the cancer cells take over. This corresponds to the lower level of p27 in Table 3. When $k_{5} / k_{5}^{\prime}=0.6$ (Figure 17(b)), the stationary state is coexistence between the two species. If $k_{5} / k_{5}^{\prime}=0.7$ we observe that cancer cells disappear and normal cells take over. This latter case is the one in which a higher $\bar{z}$ is obtained.

Figures $17(\mathrm{~d}),(\mathrm{e})$, and (f) show the spatial distribution of p27 in cancer cells for $k_{5} / k_{5}^{\prime}=0.2, k_{5} / k_{5}^{\prime}=0.6$, and $k_{5} / k_{5}^{\prime}=0.7$, respectively. We see from these plots that the concentration of p27 is lower for small values of $k_{5} / k_{5}^{\prime}$. Figures 18(a) and (b) show the number of divisions undergone by each cell for $k_{5} / k_{5}^{\prime}=0.2$ and $k_{5} / k_{5}^{\prime}=0.6$, respectively. This can be interpreted as a map of the mitotic activity of the system. ${ }^{9}$ If we compare Figures 17(d) and (e) to Figures 18(a) and (b), respectively, we can see that regions where the mitotic activity is higher correlate with regions where levels of p27 are lower. We can also see by comparing Figures 18(a) to 18(b) that the mitotic activity is higher in the first case (smaller $k_{5} / k_{5}^{\prime}$, i.e., smaller $\bar{z}$, according to Table 3 ).

\footnotetext{
${ }^{9}$ Recall that the number of divisions a given cell has undergone is given by (5.10) and is stored as the component $s_{2}(\mathbf{r})$ of the state vector.
} 


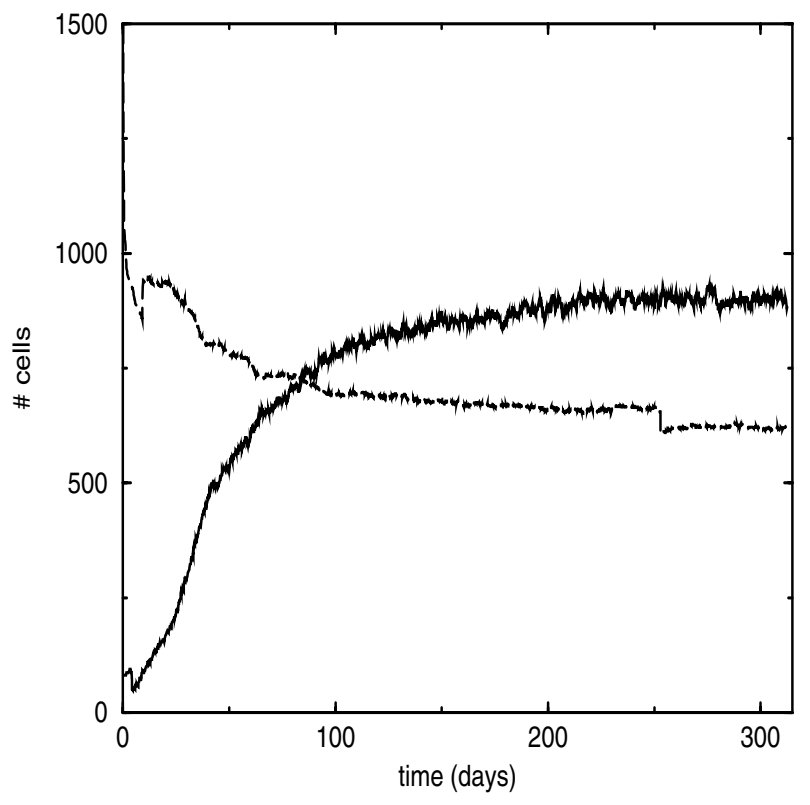

FIG. 15. Time evolution populations of normal and cancer cells in the simulation of invasion. The dashed line corresponds to normal cells, whereas the solid line corresponds to cancer cells. $k_{5} / k_{5}^{\prime}=0.2$.

Hence, we can see that the change of behavior of our model brought about by increased $k_{5} / k_{5}^{\prime}$ is, at least in part, related to a reduced proliferative capacity of the cancer cells when this parameter ratio is increased. While this agrees with the observations made in [43], it is not entirely due to a reduced proliferative capacity. If we compare Figures $8(\mathrm{a})$ and (b), we see that when $k_{5} / k_{5}^{\prime}$ increases from 0.2 to 0.7 the proliferation rate of the cancer cells becomes comparable to that of the normal cells but not much bigger. However, in [4] we have shown that increasing $k_{5} / k_{5}^{\prime}$ also increases the tendency to quiescence. On the one hand, this decreases the number of proliferating cancer cells. On the other hand, some of these quiescent cells will revert to the proliferative state if the external conditions change appropriately, while others will eventually die. As a result, the apoptosis rate of cancer cells is increased by overexpression of p27. This is also in agreement with the results of [43].

8. Growth laws. We now investigate the growth laws exhibited by our model. This is important since growth laws are used for determining the clinical course of a malignancy and designing more efficient treatment protocols [19]. In this section we show that the usual laws deduced from standard population dynamics models (e.g., logistic or Gompertz), which have been already shown to be not completely accurate [35], do not reproduce the behavior observed in cancer colonies evolving according to our model. However, the dynamics of colonies of normal cells fits quite well with the Gompertz law.

The Gompertz law arises from a population dynamics model in which the growth rate is proportional to the population size and the decay rate depends exponentially on it. The solution for such a model is given by

$$
N_{G}(t)=K \exp \left(-\lambda e^{-\frac{r t}{\ln K}}\right),
$$


(a)

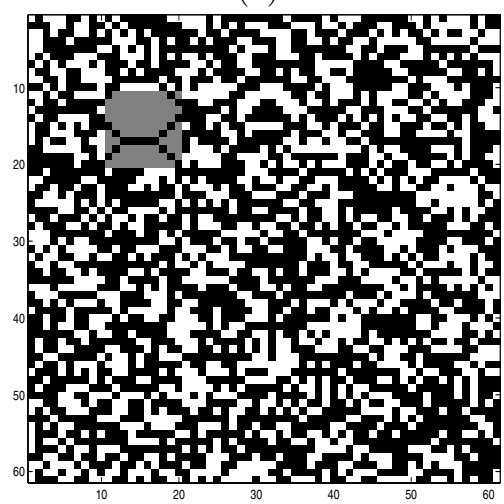

(c)

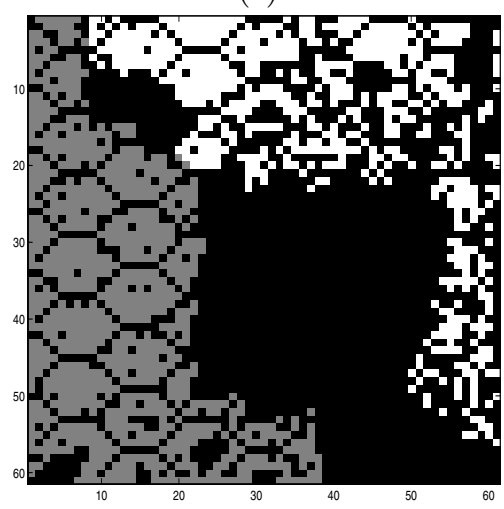

(b)

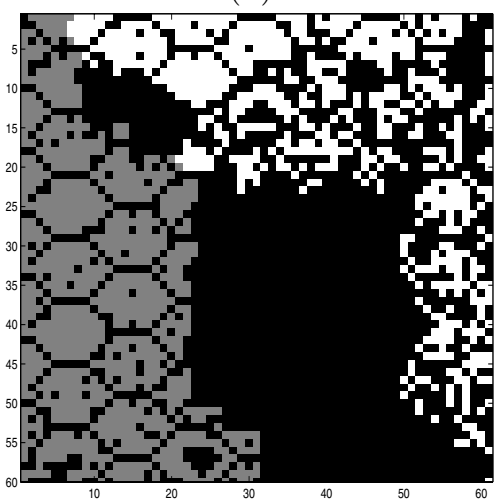

(d)

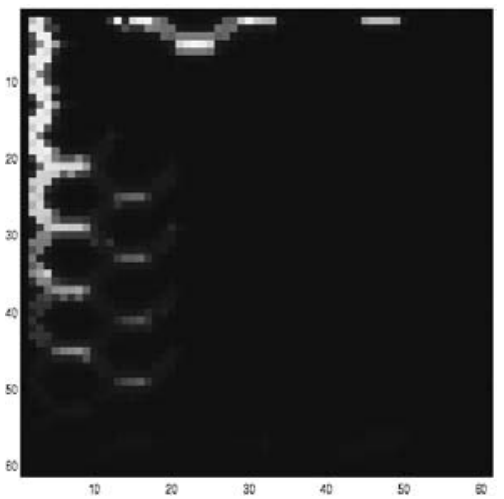

FIG. 16. (a), (b), and (c) show three snapshots of the spatial distribution of normal (white) and cancer (grey) cells for an invasion simulation, corresponding to 0, 100, and 310 days, respectively. Black elements are either empty or occupied by vessels. (d) shows the corresponding distribution of oxygen. In (d) the greyscale corresponds to the concentration of oxygen: lighter grey corresponds to higher oxygen levels.

where $K$ is the carrying capacity, $r$ is the growth rate, $\lambda=\ln \frac{N_{0}}{K}$, and $N_{0}$ is the initial population size. Therefore the quantity defined by

$$
L_{G} \equiv \ln \left(-\frac{\ln \left(N_{G}(t) / K\right)}{\ln \left(N_{0} / K\right)}\right)=-\frac{r}{\ln K} t
$$

depends linearly on $t$. We use $L_{G}$ as a reference to determine whether a population is growing according to the Gompertz law.

8.1. One-species colonies. We have carried out simulations for colonies containing only one species, either normal or cancer cells, and plotted the corresponding $L_{G}$. Figure 19(a) shows the quantity $L_{G}$ for a colony of cancer cells. We can see that $L_{G}$ does not depend linearly on time as we would expect for a population evolving according to the Gompertz law. On the other hand, Figure 19(b) shows that the behavior of a colony of normal cells may be well approximated by the Gompertz law.

8.2. Two-species colonies. With two-species colonies we have different situations to consider. First we consider values of $k_{5} / k_{5}^{\prime}$ for which the cancer cells take over. In Figure 19(c) we plot the quantity $L_{G}$ for the cancer cells (the only long-term 
(a)

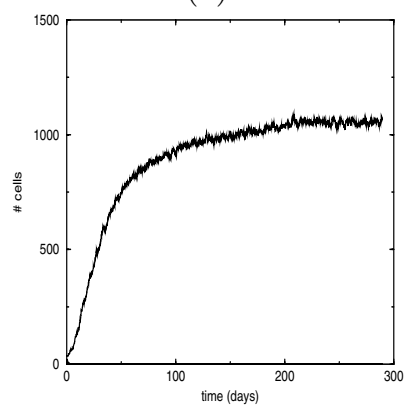

(d)

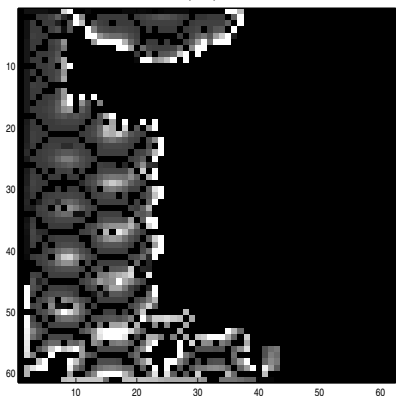

(b)

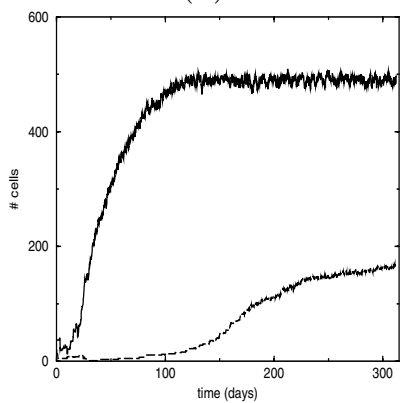

(e)

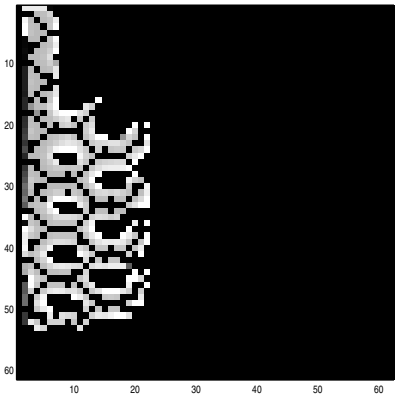

(c)

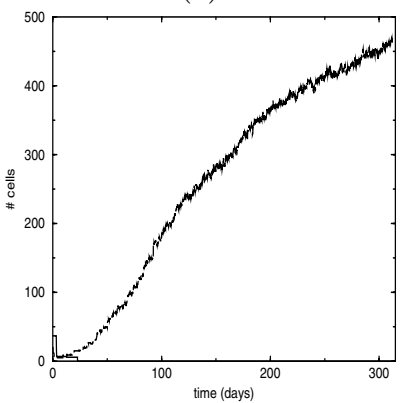

(f)

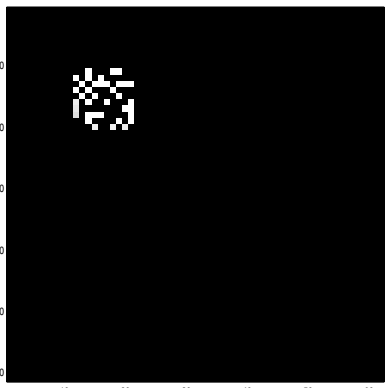

FIG. 17. (a), (b), and (c) show the time evolution of the size of the colonies for $k_{5} / k_{5}^{\prime}=0.2$, $k_{5} / k_{5}^{\prime}=0.6$, and $k_{5} / k_{5}^{\prime}=0.7$, respectively. Solid lines correspond to cancer cells and dashed lines to normal cells. (d), (e), and (f) show the corresponding pattern of concentration of $p 27$ in cancer cells. Lighter grey correspond to higher concentration of $p 27$. We can see that when the ratio $k_{5} / k_{5}^{\prime}$ grows the system changes its behavior dramatically: for lower values of $k_{5} / k_{5}^{\prime}$, cancer cells take over, whereas for high values of $k_{5} / k_{5}^{\prime}$, normal cells take over (panels (a), (b), and (c)). Concomitantly, an increase in the concentration of $p 27$ is observed when $k_{5} / k_{5}^{\prime}$ increases (panels (d), (e), and (f)). In (d) and (e) the pattern of expression of $p 27$ in cancer cells is plotted after 310 days. In (f) we show the same pattern after 30 days.

(a)

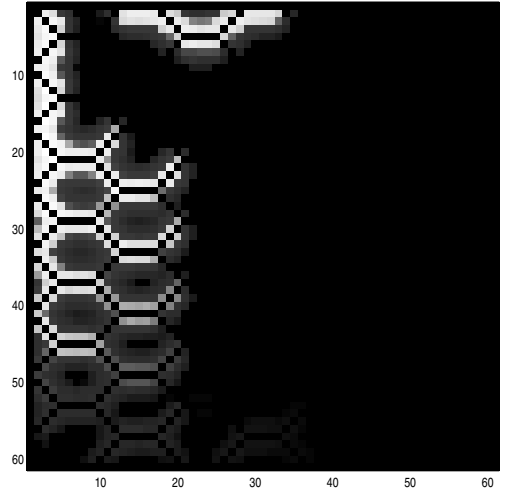

(b)

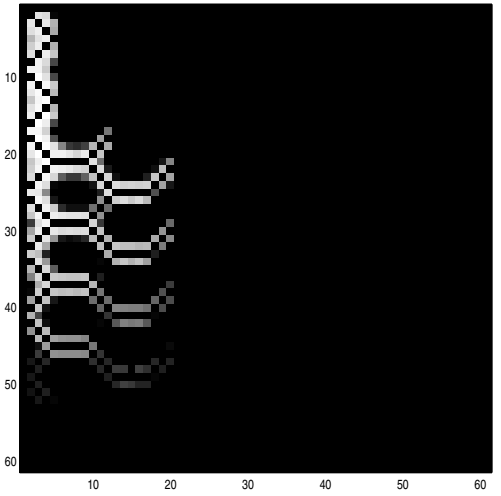

FIG. 18. Plots showing the mitotic activity for (a) $k_{5} / k_{5}^{\prime}=0.2$ and (b) $k_{5} / k_{5}^{\prime}=0.6$ after 310 days. Lighter grey corresponds to higher mitotic activity. Comparing (a) to Figure 17(d) and (b) to Figure 17(e), we can see that there is a correlation between high mitotic activity and low concentrations of $p 27$ and vice versa. 
(a)

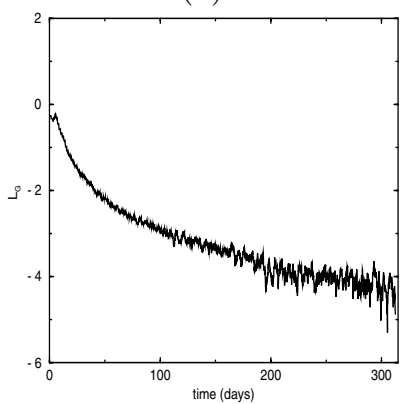

(d)

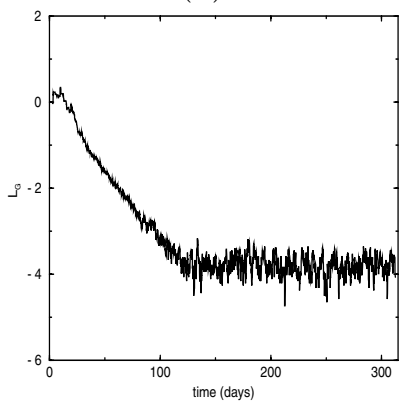

(b)

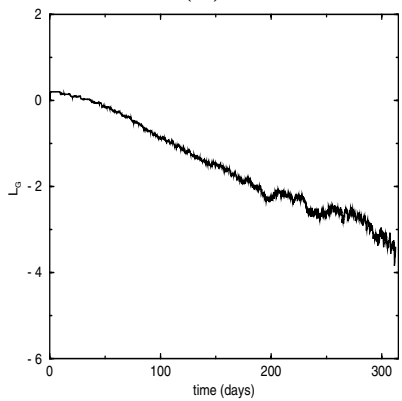

(e)

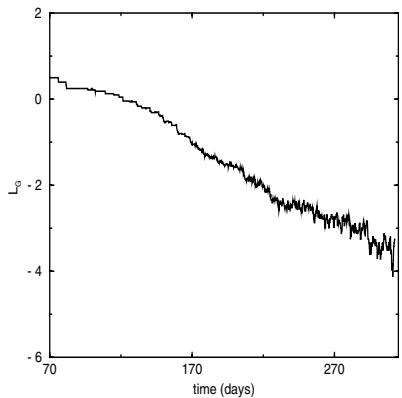

(c)

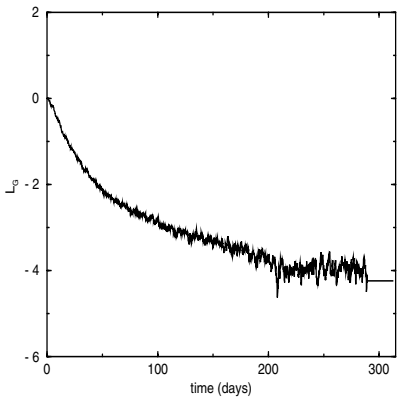

(f)

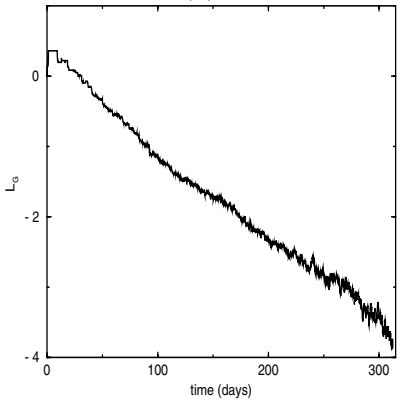

FIG. 19. $L_{G}$ for colonies of (a) cancer cells and (b) normal cells. $N_{0}=82$ for both cases. $K=1100$ for cancer cells (a) and $K=500$ for normal cells (b). In plot (c) we show $L_{G}$ for cancer cells in a two-species colony with $k_{5} / k_{5}^{\prime}=0.2 . N_{0}=37$ for normal cells and $N_{0}=20 . K=1125$ for cancer cells. $L_{G}$ for (d) cancer cells and (e) normal cells in a two-species colony with $k_{5} / k_{5}^{\prime}=0.6$. $N_{0}=37$ for normal cells and $N_{0}=20 . K=520$ for cancer cells (d) and $K=175$ for normal cells (e). Plot (f) shows $L_{G}$ for normal cells in a two-species colony with $k_{5} / k_{5}^{\prime}=0.7 . N_{0}=37$ for normal cells and $N_{0}=20 . K=500$ for normal cells.

survivors in this particular case). As in Figure 19(a) the behavior is not Gompertzlike.

The second case corresponds to values of $k_{5} / k_{5}^{\prime}$ such that there is long-term coexistence between normal and cancer cells (Figures 19(d) and (f)). Once again, the cancer cells do not behave as predicted by the Gompertz law (Figure 19(d)). However, the long-term behavior of the normal cells is well described by a Gompertz law (Figure 19(e)).

The last situation corresponds to values of $k_{5} / k_{5}^{\prime}$ such that the normal cells take over. In this case (see Figure 19(f)) the dynamics of $L_{G}$ for the normal cells (the long-term survivors in this case) fits very well to a Gompertz law.

8.3. Growth laws for cancer cells. The results presented above seem to confirm that, in our model, a colony of normal cells in an inhomogeneous environment grows according to the Gompertz law. However, the cancer cells appear to behave in a different way:

$$
N(t)=\left\{\begin{array}{l}
C_{1} \exp (-r t) \text { if } t \leq t_{c r}, \\
C_{2} t^{\delta} \text { if } t_{c r} \leq t \leq t_{s a t} \\
C_{3} \text { if } t \geq t_{\text {sat }},
\end{array}\right.
$$

where $C_{1}, C_{2}, C_{3}, r$, and $\delta$ are constants. Figures 20(a) and (b) and Figures 20(c) and (d) illustrate (8.3) for a one-species colony and a two-species colony, respectively. 
(a)

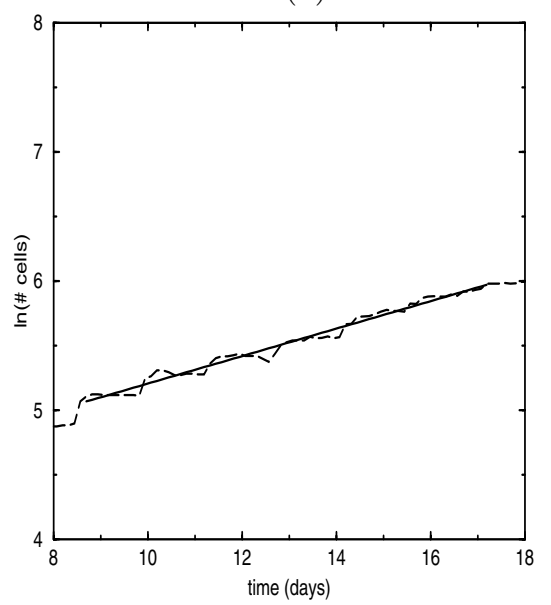

(c)

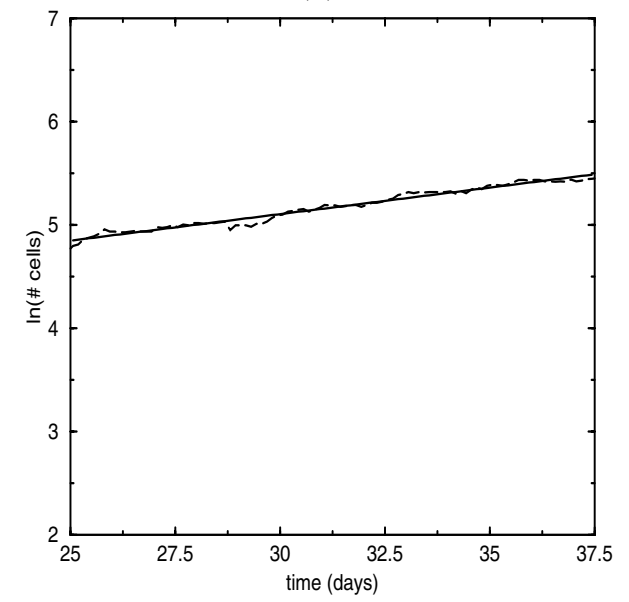

(b)

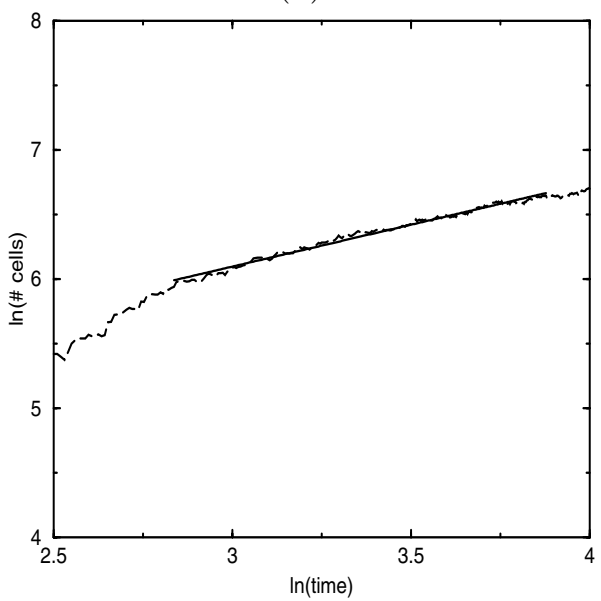

(d)

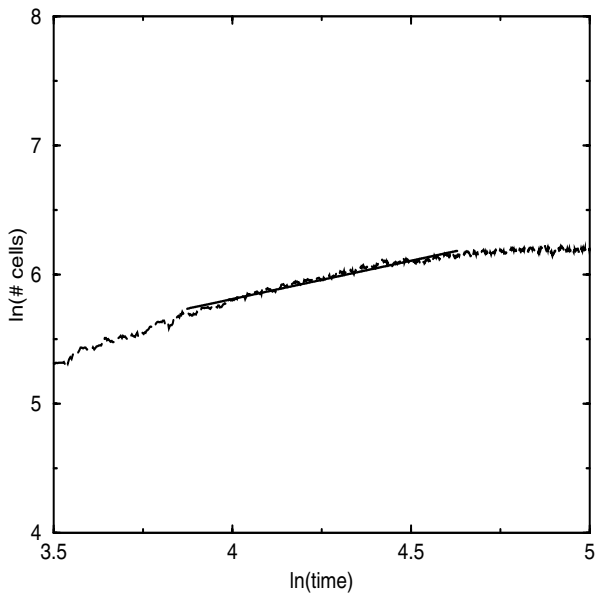

FIG. 20. (a) Semilogarithmic plot of the initial phase of a one-species (cancer) colony. This plot shows that, initially, the colony grows exponentially. The growth rate is $r=0.106 d_{a y}^{-1}$. (b) Loglog plot at a later stage in the progression of the same colony, which shows how the growth changes from exponential to algebraic. The exponent $\delta=0.66$. In both cases the correlation coefficient of the linear regression is larger than 0.99. Solid lines correspond to the fitted curve and dashed lines to the results of our simulations. (c) Semilogarithmic plot of the initial phase of a two-species colony. This plot shows that, initially, cancer cells grow exponentially. The growth rate is $r=0.051 d^{2} y^{-1}$. (d) Log-log plot of a later stage in the progression of the same colony, which shows how the growth of the cancer cells change from exponential to algebraic. $\delta=0.61$. In both cases the correlation coefficients of the linear regression is larger than 0.99. Solid lines correspond to the fitted curve and dashed lines to the results of our simulations.

Figures 20(a) and 20(c) show that the growth of the colony (in both cases) is approximately exponential at early times. Figures 20(b) and 20(d) show that at late times the growth of the colonies can be described by an algebraic law. Our estimates of $r$ and $\delta$, which give an indication of how fast the growth is, are smaller in the case of the two-species colony due to competition (see the caption of Figure 20).

These results are in qualitative agreement with experimental [11] and simulation [21] results for avascular tumors. The off-lattice simulations reported in [21] are carried out using a Monte Carlo method. In these simulations cell-to-cell (mechanical) 
interactions are accounted for by a model potential energy. Cells are supposed to be perfect spheres at the beginning of the cell cycle and deform into a dumbbell shape as cells enter mitosis. This deformation modifies the total energy of the system, and the neighboring cells respond by changing their position or orientation in order to minimize the total energy. Neither in the experiments nor in the simulations were nutrient levels and its spatial distribution taken into account. In these two papers algebraic growth is reported for tumor cells growing in vitro. The exponents obtained in $[11,21](\delta \sim 3)$ are different from the ones we obtain from our model. Better quantitative agreement is obtained with the results of the study presented in [35]. In [35] the authors analyzed the growth law of primary breast cancer from mammography screening data, obtaining an algebraic growth law, rather than a Gompertz-like growth as is usually assumed, with an exponent $\delta \sim 0.5$.

Since both normal and cancer cells evolve in a qualitatively similar environment, and the rules for cell-to-cell interaction are the same in both cases, the reasons for their different temporal behavior must be found in the intracellular dynamics and, in particular, their cell-cycle dynamics. From this point of view, there are two major differences between normal and cancer cells: cancer cells divide at a higher rate than normal cells and cancer cells may become quiescent, whereas normal cells may not. However, for the situation shown in Figure 17(b), the proliferation rates of the normal and cancer cells are similar, as we can see from Figure 8. Therefore, the different behavior of the cancer cells must be due to their ability to delay death by starvation by entering a quiescent state.

9. Discussion. In this paper we have proposed a multiple scale model for vascular tumor growth in which we have integrated phenomena at the tissue scale (vascular structural, adaptation, and blood flow), cellular scale (cell-to-cell interaction), and the intracellular scale (cell-cycle, VEGF production, and apoptosis). To the best of our knowledge, this is the first example of such a model for tumor development.

Our integrative approach allows us to analyze the effect on the population of changes in the intracellular processes of its components. The results produced by our model are in agreement with clinical studies highlighting the p27 protein as a prognostic indicator. Furthermore, our model suggests that the p27 protein may be considered a therapeutic target. Guided by the results of section 7.2 , we conclude that p27 expression could be used to reduce the competitive advantage of the tumor cells over the normal cells. This could be achieved by altering the normal pattern of regulation of $\mathrm{p} 27$. However, in our model the dynamics of the $\mathrm{p} 27$ protein have been modeled simply. A more accurate model would be required to test our hypothesis.

Another issue we have addressed is to which growth laws our model gives rise. We have shown that the population of normal cells evolve according to a Gompertz law. This is an emergent property of our model: we have studied the evolution of a colony of cells under given environmental conditions and internal division dynamics. We have nowhere imposed that the death depends exponentially on the size of the colony. On the other hand, we have shown that the Gompertz law fails to reproduce the behavior of the cancer cells. Instead, the colonies of cancer cells go through three different regimes: exponential growth, algebraic growth, and, finally, saturation. This difference between the two populations is due to the ability of the cancer cells to enter a quiescent state before dying. ${ }^{10}$ Further mathematical analysis of populations

\footnotetext{
${ }^{10}$ In fact, we have recently become aware of results obtained from continuous models which yield the same behavior: normal cell growth fits the Gompertz law, whereas cancer cells (which are assumed to enter quiescence upon starvation) do not [67].
} 
undergoing quiescence before dying is needed to confirm this point.

A weakness of our model is that the dynamics of p53 and intracellular VEGF are very simplistic. A more detailed model for their pattern of expression would be needed in order to produce more reliable results. Also, for our model of the effect of hypoxia on the cell-cycle we have built up on a model originally proposed for yeast. This model also needs further refinement.

Another area in which our model could be improved is angiogenesis. Although we have included tissue-vasculature coupling, we have not introduced new vessel formation, although it might be argued that this is not so important in naturally wellvascularized tissues, for example the liver. Concerning our treatment of the vasculature and blood flow, there are two points in which our model needs further refinement: the use of the Poiseuille law for blood flow, which is not valid in very small capillaries, and the assumption that the vessels are rigid rather than compliant tubes. However, our model takes into account some properties of real vascular networks and blood flow that allow us to assess the effects of blood flow heterogeneity. This is particularly important when trying to model drug delivery and evaluate treatment protocols (see [61] for a particular example).

There are, of course, many other factors that should be included in a more accurate description of the carcinogenic process. For example, we have assumed that cancer cells do not migrate, whereas, in practice, cancer cells usually acquire a metastatic phenotype which enables them to migrate and metastasize to other tissues close to the primary tumor. Another important factor that should be explored concerns interactions between the tumor and the immune system: in the early stages of tumor development, when cancer cells have not yet condensed to form a solid tumor, (individual) malignant cells may be recognized by the immune system and removed [9]. Knowledge of the mechanisms by which the immune response is activated by the presence of tumor cells and, especially, the reasons for the failure of immune surveillance are issues that might prove important in the development of new treatments.

Another (rather technical) shortcoming of our model concerns its application to a more developed stage of tumor growth, where the tumor is likely to comprise millions of cells rather than thousands of cells, as has been considered in the present study. In such a case we would need to resort to parallel computing techniques. Although this certainly implies some degree of complication from the computational point of view, the present model should not be too difficult to implement in parallel, as the intracellular dynamics, which is the most time-consuming part, is completely local (the intracellular dynamics of different elements are only coupled "indirectly" by the global fields (oxygen, VEGF), with no direct interaction between them). A threedimensional version of this model is another issue that would involve the use of more refined numerical and computational techniques. The major problem would be solving the reaction-diffusion equations for the global fields (e.g., oxygen or VEGF) in three dimensions. A possible solution might be to formulate these reaction-diffusion phenomena in terms of a lattice gas (as in [20]) and using parallel computing techniques.

Many other issues, such as different boundary conditions for the diffusion of oxygen and VEGF and different boundary conditions for the vascular network, could have been explored. However, in the present article our main aim was to develop our modeling framework and present some examples of biologically and clinically relevant issues that can be addressed using this framework.

Most of these limitations will be dealt with in future research.

Finally, our model might also give some insight into the controversy surrounding 
Folkman's model for tumor therapy: disrupting angiogenesis yields a starving tumor which eventually dies. In our model, disruption of angiogenesis (by the introduction of some VEGF-specific antibody), would eliminate the coupling between the growing tumor and the vasculature. This would not eliminate the tumor (see Figures 12(d), (e), and (f) and Figures 12(g), (h), and (i)), although it would reduce its size. This result suggests that combination with other therapies will be necessary for a successful treatment of vascular tumors.

Acknowledgment. TA thanks the Centre for Mathematical Biology, University of Oxford, where most of this work was done.

\section{REFERENCES}

[1] J. A. AdAm, A mathematical model of tumour growth II: Effects of geometry and spatial uniformity on stability, Math. Biosci., 86 (1987), pp. 183-211.

[2] J. A. Adam, A mathematical model of tumour growth III: Comparison with experiment, Math. Biosci., 86 (1987), pp. 213-227.

[3] T. Alarcón, H. M. Byrne, and P. K. Maini, A cellular automaton model for tumour growth in inhomogeneous environment, J. Theor. Biol., 225 (2003), pp. 257-274.

[4] T. Alarcón, H. M. Byrne, And P. K. Maini, Mathematical model of the effect of hypoxia on the cell-cycle of normal and cancer cells, J. Theor. Biol., 229 (2004), pp. 395-411.

[5] M. S. Alber, M. A. Kiskowski, J. A. Glazier, and Y. JiAng, On cellular automaton approaches to modeling biological cells, in Mathematical Systems Theory in Biology, Communications, Computation, and Finance, J. Rosenthal and D. S. Gilliam, eds., IMA Vol. Math. Appl. 134, Springer-Verlag, New York, 2003, pp. 1-39.

[6] B. Alberts, D. Bray, J. Lewis, M. Raff, K. Roberts, and J. D. Watson, Molecular Biology of the Cell, 3rd ed., Wiley-Interscience, New York, 1994.

[7] A. R. A. Anderson and M. A. J. Chaplain, Continuous and discrete mathematical models of tumor-induced angiogenesis, Bull. Math. Biol., 60 (1998), pp. 857-899.

[8] S. A. Barber, Soil Nutrient Bioavailability: A Mechanistic Approach, Garland, New York, 1984.

[9] N. Bellomo and L. Preziosi, Modelling and mathematical problems related to tumour evolution and its interaction with the immune system, Math. Comput. Modelling, 32 (2000), pp. $413-452$.

[10] C. J. W. Breward, H. M. Byrne, and C. E. Lewis, A multiphase model describing vascular tumour growth, Bull. Math. Biol., 65 (2003), pp. 609-640.

[11] A. Bru, J. M. Pastor, I. Fernaud, I. Bru, S. Melle, and C. Berenguer, Super-rough dynamics on tumor growth, Phys. Rev. Lett., 81 (1998), pp. 4008-4011.

[12] H. M. Byrne And M. A. J. Chaplain, Free boundary value problems associated with the growth and development of multicellular spheroids, European J. Appl. Math., 8 (1997), pp. 639-658.

[13] H. M. Byrne, J. R. King, And D. L. S. McElwain, A two-phase model of solid tumour growth, Appl. Math. Lett., 16 (2003), pp. 567-573.

[14] P. Carmeliet, Y. Dor, J.-M. Herbert, D. Fukumura, K. Brusselmans, M. Dewerchin, M. Neeman, F. Bono, R. Abramovitch, P. Maxwell, K. Koch, P. Ratcliffe, L. Moons, R. Jain, D. Collen, And E. Keshet, Role of HIF- $\alpha$ in hypoxia-mediated apoptosis, cell proliferation and tumour angiogenesis, Nature, 394 (1998), pp. 485-490.

[15] M. A. J. Chaplain, Avascular growth, angiogenesis and vascular growth in solid tumours: The mathematical modelling of the stages of tumour development, Math. Comput. Modelling, 23 (1996), pp. 47-87.

[16] R. Chatuverdi, J. A. Izaguirre, C. Huang, T. Cickovski, P. Virtue, G. Thomas, G. Forgacs, M. Alber, G. Hentschel, S. A. Newman, and J. A. Glazier, Multi-model simulations of chicken limb morphogenesis, in International Conference on Computational Science, Lecture Notes in Comput. Sci. 2659, Springer-Verlag, Berlin, 2003, pp. 39-49.

[17] J. L. Cherry and F. R. Adler, How to make a biological switch, J. Theor. Biol., 203 (2000), pp. 117-133.

[18] S. Cory, D. C. S. Huang, And J. M. Adams, The Bcl-2 family: Roles in cell survival and oncogenesis, Oncogene, 22 (2003), pp. 8590-8607.

[19] J. Crown, High dose chemotherapy of metastatic breast cancer: The end of the beginning?, British J. Cancer, 75 (1997), pp. 467-469. 
[20] A. Deutsch And S. Dormann, Modeling of avascular tumor growth with a hybrid cellular automaton, In Silico Biol., 2 (2002), pp. 1-14.

[21] D. Drasdo And S. Hohme, Individual-based approaches to birth and death in avascular tumors, Math. Comput. Modelling, 37 (2003), pp. 1163-1175.

[22] J. Folkman And M. Hochberg, Self-regulation of growth in three dimensions, J. Exp. Med., 138 (1973), pp. $745-753$.

[23] S. J. Franks, H. M. Byrne, J. R. King, and C. E. Lewis, Modelling the growth of ductal carcinoma in situ, J. Math. Biol., 47 (2003), pp. 424-452.

[24] Y. C. Fung, Biomechanics, Springer-Verlag, New York, 1993.

$[25]$ J. O. Funk, Cancer cell cycle control, Anticancer Res., 19 (1999), pp. 4772-4780.

[26] D. Gammak, H. M. Byrne, And C. E. Lewis, Estimating the selective advantage of mutant p53 tumour cells to repeated rounds of hypoxia, Bull. Math. Biol., 19 (1999), pp. 4772-4780.

[27] L. B. Gardner, Q. Li, M. S. Park, W. M. Flanagan, G. L. Semenza, and C. V. Dang, Hypoxia inhibits $\mathrm{G}_{1} / \mathrm{S}$ transition through regulation of $p 27$ expression, J. Biol. Chem., 276 (2001), pp. 7919-7926.

[28] R. A. GATENBY, Application of competition theory to tumour growth: Implications for tumour biology and treatment, Eur. J. Cancer., 32A (1996), pp. 722-726.

[29] R. A. Gatenby and E. T. Gawlinski, A reaction-diffusion model of cancer invasion, Cancer Res., 56 (1996), pp. 5745-5753.

[30] D. Goldman And A. S. Popel, A computational study of the effect of capillary network anastomoses and tortuosity on oxygen transport, J. Theor. Biol., 206 (2000), pp. 181-194.

[31] T. G. Graeber, C. Osmanian, T. Jacks, D. E. Housman, C. J. Koch, S. W. Lowe, and A. J. Giaccia, Hypoxia-mediated selection of cells with diminished apoptotic potential in human tumours, Nature, 379 (1996), pp. 89-91.

[32] F. Graner and J. A. Glazier, Simulation of biological cell sorting using a two-dimensional extended Potts model, Phys. Rev. Lett., 69 (1992), pp. 2013-2016.

[33] H. P. Greenspan, Models for the growth of a solid tumour by diffusion, Stud. Math. Appl., 51 (1972), pp. 317-340.

[34] H. P. Greenspan, On the growth and stability of cell cultures and solid tumours, J. Theor. Biol., 56 (1976), pp. 229-242.

[35] D. HaRt, E. Scochat, AND Z. Agur, The growth law of primary breast cancer as inferred from mammography screening trial data, British J. Cancer, 78 (1998), pp. 382-387.

[36] P. J. Hunter, P. Robbins, and D. Noble, The IUPS human physiome project, Pflügers Archiv- Eur. J. Physiol., 445 (2002), pp. 1-9.

[37] P. J. Hunter, P. Kohl, And D. Noble, Integrative models of the heart: Achievements and limitations, Philos. Trans. Roy. Soc. London Ser. A, 359 (2001), pp. 1049-1054.

[38] T. Jackson and H. M. Byrne, A mathematical model of tumour encapsulation, Math. Biosci., 180 (2002), pp. 307-328.

[39] R. K. JAIn, Delivery of molecular and cellular medicine to solid tumours, Advanced Drug Delivery Reviews, 46 (2001), pp. 149-168.

[40] A. R. Kansal, S. Torquato, G. R. Harsh, IV, E. A. Chiocca, and T. S. Deisboeck, Simulated brain tumour growth dynamics using a three-dimensional cellular automaton, J. Theor. Biol., 203 (2000), pp. 367-382.

[41] F. D. L. Kelpin, M. A. Kirkilionis, And B. W. Kooi, Numerical methods and parameter estimation of a structured population model with discrete events in the life history, J. Theor. Biol., 207 (2000), pp. 217-230.

[42] K. W. Kinzler and B. Vogelstein, Life (and death) in a malignant tumour, Nature, 379 (1996), pp. 19-20.

[43] R. M. Kirla, H. K. Haapasalo, H. Kalimo, and E. K. Salminen, Low expression of p27 indicates a poor prognosis in patients with high grade astrocytomas, Cancer, 97 (2003), pp. 644-648.

[44] M. A. Konerding, E. Fait, And A. Gautmann, 3D microvascular architecture of precancerous lesions and invasive carcinomas, British J. Cancer, 84 (2001), pp. 1354-1362.

[45] H. A. Levine, S. Pamuk, B. D. Sleeman, and M. Nilsen-Hamilton, Mathematical modeling of capillary formation and development in tumor angiogenesis: Penetration into the stroma, Bull. Math. Biol., 63 (2001), pp. 801-863.

[46] B. P. Marchant, J. Norbury, and J. A. Sherratt, Travelling wave solutions to a haptotaxisdominated model of malignant invasion, Nonlinearity, 14 (2001), pp. 1653-1671.

[47] D. L. S. McElwain And L. E. Morris, Apoptosis as a volume loss mechanims in mathematical models of tumour growth, Math. Biosci., 39 (1978), pp. 147-157.

[48] J. Moreira And A. Deutsch, Cellular automaton models of tumour development: A critical review, Adv. Complex Sys., 5 (2002), pp. 247-267. 
[49] W. K. Nisioka And R. M. Welsh, Susceptibility to cytotoxic T lymphocite-induced apoptosis is a function of the proliferative status of the target, J. Exp. Med., 179 (1994), pp. 769-774.

[50] P. H. Nye And P. B. Tinker, Solute Movement in the Soil-Root System, Blackwell Science, Oxford, UK, 1977.

[51] M. E. Orme And M. A. J. Chaplain, A mathematical model of vascular tumour growth and invasion, Math Comput. Modelling, 23 (1996), pp. 43-60.

[52] A. A. Patel, E. T. Gawlinski, S. K. Lemieux, and R. A. Gatenby, A cellular automaton model of early tumor growth and invasion: The effects of native tissue vascularity and increased anaerobic tumor metabolism, J. Theor. Biol., 213 (2001), pp. 315-331.

[53] G. Pettet, C. P. Please, M. J. Tindall, and D. L. S. McElwain, The migration of cells in multicell tumor spheroids, Bull. Math. Biol., 63 (2001), pp. 231-257.

[54] C. P. Please, G. Pettet, and D. L. S. McElwain, A new approach to modelling the formation of necrotic regions in tumours, Appl. Math. Lett., 11 (1998), pp. 89-94.

[55] M. J. Poole, A. V. Holden, And J. V. Tucker, Hierarchical reconstruction of cardiac tissue, Chaos Solitons Fractals, 13 (2002), pp. 1581-1612.

[56] W. H. Press, S. A. Teukolsky, W. T. Vetterling, and B. P. Flannery, Numerical Recipes in $C$, Cambridge University Press, Cambridge, UK, 1992.

[57] L. Preziosi, Ed., Cancer Modelling and Simulation, CRC Press, Boca Raton, FL, 2003.

[58] A. R. Pries, T. W. Secomb, T. Gessner, M. B. Sperandio, J. F. Gross, and P. Gaehtgens, Resistance to blood flow in microvessels in vivo, Circ. Res., 75 (1994), pp. 904-915.

[59] A. R. Pries, T. W. Secomb, and P. Gaehtgens, Design principles of vascular beds, Circ. Res., 77 (1995), pp. 1017-1023.

[60] A. R. Pries, T. W. Secomb, and P. Gaentgens, Structural adaptation and stability of microvascular networks: Theory and simulations, Am. J. Physiol., 275 (1998), pp. H349H360.

[61] B. Ribba, K. Marron, Z. Agur, T. Alarcón, and P. K. Maini, Doxorubicin treatment efficacy on non-Hodgkin's lymphomas: Computer model and simulations, Bull. Math. Biol., submitted.

[62] T. Roose, A. C. Fowler, And P. R. Darrah, A mathematical model of plant nutrient uptake, J. Math. Biol., 42 (2001), pp. 347-360.

[63] D. H. Rothman and S. Zalesky, Lattice-Gas Cellular Automata: Simple Models of Complex Hydrodynamics, Cambridge University Press, Cambridge, UK, 1997.

[64] J. A. Royds, S. K. Dower, E. E. Qwarstrom, And C. E. Lewis, Response of tumour cells to hypoxia: Role of p53 and NFkB, J. Clin. Path.: Mol. Pathol., 51 (1998), pp. 55-61.

[65] Z. Sinik, T. Alkibay, O. Ataoglu, H. Biri, S. Sozen, N. Dinez, U. Karaoglan, and I. Bozkirli, Nuclear p53 overexpression in bladder, prostate, and renal carcinomas, Int. J. Urol., 4 (1997), pp. 546-551.

[66] N. P. Smith, P. J. Mulquiney, M. P. Nash, C. P. Bradley, D. P. Nickerson, and P. J. Hunter, Mathematical modelling of the heart: Cell to organ, Chaos Solitons Fractals, 13 (2002), pp. 1613-1621.

[67] M. J. Tindall, Modelling Cell Movement and the Cell Cycle in Multicellular Tumour Spheroids, Ph.D. dissertation, University of Southampton, Southampton, UK, 2002.

[68] S. Turner AND J. A. Sherrat, Intercellular adhesion and cancer invasion: A discrete simulation using the extended Potts model, J. Theor. Biol., 216 (2002), pp. 85-100.

[69] J. J. Tyson AND B. NovaK, Regulation of the eukariotic cell cycle: Molecular antagonism, hysteresis, and irreversible transitions, J. Theor. Biol., 210 (2001), pp. 249-263.

[70] J. Valenciano And M. A. J. Chaplain, An explicit subparametric spectral element method of lines applied to a tumour angiogenesis system of partial differential equations, Math. Models Methods Appl. Sci., 14 (2004), pp. 165-187.

[71] J. P. WARD AND J. R. KING, Mathematical modelling of avascular-tumour growth, IMA J. Math. Appl. Med., 14 (1997), pp. 39-69.

[72] J. P. WARD AND J. R. KING, Mathematical modelling of avascular-tumour growth II: Modelling growth saturation, IMA J. Math. Appl. Med., 16 (1999), pp. 171-211. 